\title{
A four-quadrant mobility model-based routing protocol for post-earthquake emergency communication network
}

\author{
Xiaoming Wang ${ }^{1,2}$ (i) and Chang Guo ${ }^{3,4^{*}}$ (i)
}

\section{${ }^{*}$ Correspondence:}

guochang@mail.dhu.edu.cn

${ }^{3}$ The College of Information,

Mechanical, and Electrical

Engineering, Shanghai

Normal University, Shanghai, China

Full list of author information is available at the end of the article

\begin{abstract}
Emergency communication network (ECN) is the important infrastructure to acquire the real-time information after disaster, which is essential for rescue task. However, the existed routing protocols seldom consider the uneven distribution of rescue area so that cannot satisfy the ECN's requirement and the quality of rescue needs improvement. In this paper, we avoided the traditional linear propulsion from rim to core and proposed a novel four-quadrant mobility model (FQMM), which makes rescuers arrive the most intensity core area first, which can improve the rescue quality. Then, a FQMMbased protocol (FQMMBP) for ECN is designed, which aims to improve the performance of ECN in terms of package delivery rate (PDR) and end-to-end delay. Finally, we set up a virtual earthquake scenario to simulate our proposed protocol in NS2. Results show that the proposed protocol outperforms to the three compared routing protocols, i.e., AODV, DSDV and DSR. The average FDR is improved by $16.31 \%$, and the average reduction of delay is $64.45 \%$, which shows our proposed scheme's advantages in quality of rescue.
\end{abstract}

Keywords: ECN, FQMM, FQMMBP, Rescue urgency degree, Post-earthquake

\section{Introduction}

\subsection{Background}

After the devastating earthquake, emergency rescue crews are needed to rush to the scene for rescue as soon as possible. Post-earthquake emergency rescue includes material dispatching, team dispatching, personnel evacuation, post-disaster reconstruction, etc. Due to the different catastrophic degrees caused by the earthquake in different affected areas, the rescue urgency degrees (RUDs) are different for those areas. Seismic intensity, which refers to the catastrophic degree of earthquake impact on the ground or artificial buildings in a certain area [1], can be used to express the rescue urgencies of disaster areas. The seismic intensity can be obtained in the following ways [2]: In the area where seismic observation equipment is intensively deployed, we can directly obtain the intensity distribution map of the instrument; In the area where seismic observation equipment is sparsely deployed, we can obtain the intensity distribution map of the instrument by grid interpolation; In the area where seismic observation equipment author(s) and the source, provide a link to the Creative Commons licence, and indicate if changes were made. The images or other third party material in this article are included in the article's Creative Commons licence, unless indicated otherwise in a credit line to the material. If material is not included in the article's Creative Commons licence and your intended use is not permitted by statutory regulation or exceeds the permitted use, you will need to obtain permission directly from the copyright holder. To view a copy of this licence, visit http:// creativecommons.org/licenses/by/4.0/. 
is rarely deployed, we can obtain the intensity distribution map either by historical seismic data statistics or earthquake simulations. Therefore, it is feasible to carry out emergency rescue according to the emergency degree of the disaster area reflected by seismic intensity. When seismic intensity is obtained, the degree of emergency rescue can be determined by the value of seismic intensity, e.g., after an earthquake, the degree of emergency rescue in high intensity areas is higher than that in low intensity areas, while in the region with equal seismic intensity, the degrees of emergency rescue are equal.

After the devastating earthquake, the communication infrastructure and power system in the disaster area will suffer from different degrees of damage, resulting in communication system paralysis. Rescue crews have to quickly establish ECN in the disaster area to meet the communication demands both inside and outside the affected area. Unlike traditional self-organized network, the nodes in ECN have the characteristics of energy limitation, high energy consumption in communication and low energy consumption in data calculation. In recent years, the research on ECN routing protocols has been widely concerned by scholars.

\subsection{Relate works}

In the existing research on the mobility model of emergency rescue crews, the traditional task assignment model, e.g., dynamic multi-stage allocation model [3], optimal scheduling strategy [4] and multi-objective optimization model [5], usually focuses on processing of rescue time. Song Ye et al. [6] established an optimization model for the assignment of earthquake emergency rescue teams, aiming at improving rescue efficiency and maximizing the satisfaction of rescue time. Yuan Jinsha et al. [7] considered demand urgency and satisfaction and constructed a dispatch model of multi-reserve point and multi-disaster point in two-ant group algorithm, which provides a reference to the disaster relief strategy for decision makers. Md. Ibrahim Talukdar et al. [8] investigated shortest path map based (SPMB), random way point (RWP) and random walk (RW) models to measure the efficiency of mobility models. Cao Cejun et al. [9] formulated a bi-level integer programming model (BIPM) to minimize total weighted travel time at the upper level and to maximize total weighted survivors' perceived satisfaction at the lower level, and then, a case study from Wenchuan earthquake was presented to illustrate the proposed model and solution strategies. Song Yinghua et al. [10] proposed a satisfaction function to measure the psychology of the victims. The emergency material distribution plan optimization model (EMDPOM) was established based on the satisfaction of the victims and the minimum total system cost, combined with the location problem of the emergency distribution center in the two-level distribution network. The model was solved by a real-coded genetic algorithm, and the data in the Wenchuan earthquake were taken as an example to verify the validity and feasibility of the model and algorithm. However, the above researches studied the mobility models from the perspective of rescue capacity and relief materials repertory, without considering seismic intensity in disaster areas. Furthermore, the above researches did not reasonably allocate the communication and rescue nodes based on the characteristics of ECN. Comparisons of traditional mobility models are listed in Table 1.

In order to improve the positioning accuracy of wireless sensor network (WSN) nodes, Yuan Ruilin [11] proposed a WSN node positioning algorithm that narrowed the initial 
Table 1 Comparisons of traditional mobility models

\begin{tabular}{lllll}
\hline Mobility model & $\begin{array}{l}\text { Single mobility } \\
\text { model }\end{array}$ & $\begin{array}{l}\text { Group mobility } \\
\text { model }\end{array}$ & $\begin{array}{l}\text { Suitable for } \\
\text { emergency rescue }\end{array}$ & Simple or complicated \\
\hline SPMB & Yes & No & No & Simple \\
RWP & Yes & No & No & Simple \\
RW & Yes & No & No & Simple \\
BIPM & No & Yes & Yes & Complicated \\
EMDPOM & No & Yes & Yes & Complicated \\
\hline
\end{tabular}

values of Kalman filter by improving the initial value calculation method of multi-hop positioning algorithms. Simulations showed that the novel algorithm can save computing and communication costs under emergency communication environment. In emergency communication networks, a complete end-to-end communication link between the source node and the destination node may not always be available, Li Guangqiang et al. [12] studied the intermittent connection characteristics of DTN and proposed an improved routing algorithm based on the encounter probability between nodes (RAEPBN). Simulation results showed that RAEPBN has the best performance in delivery rate, average delay and network overhead, compared with the existing Epidemic and Prophet routing algorithms. Yu Xiang et al. [13] improved ad hoc on-demand distance vector routing (AODV) protocol for emergency communication scenarios and proposed SE-AODV protocol which can reduce energy consumption and extend lifetime in ECN. Bamhdi Alwi M. [14] proposed a DP-AODV method by adapting the standard AODV protocol to dynamically adjust transmission power usage, which used the dependence of a transmission range on density. Simulation results showed that DP-AODV increased network throughput while reducing the node interference in a dense region, as well as it enhanced the overall network performance with respect to the increased packet delivery fraction, reducing the control overheads and jitter, enhancing overall throughput, reducing interferences and finally, shortening end-to-end delay in medium to high density conditions. As wireless node has a short transmission range in ECN, communication between any two partitioned networks occurs only through the node mobility. Syed Rabiya et al. [15] proposed a replica reduced and energy-based routing protocol (REB) to control the replicas and increased the packet delivery ratio in emergency scenarios. Simulation results demonstrated that REB increased delivery rate and reduced overhead and energy consumption considerably, resulting in increased life span of ECN. Data transmission requirements in ECNs are disorganized; Masud Farhan et al. [16] proposed an emergency Traffic Adaptive MAC (eTA-MAC) protocol based on Prioritization to provide traffic ranking through a traffic class prioritization-based slotted-carrier sense multiple access/collision avoidance (TCP-CSMA/CA) scheme. The results indicated that the proposed protocol is better than the existing medium access control (MAC) protocols by $86 \%$ decrease in packet delivery delay, $61 \%$ increase in throughput and a $76 \%$ decrease in energy consumption. In ECN, the routing discovery process of on-demand routing protocol is expensive, and the energy consumption of nodes is large. In order to solve the above problems, Ramalakshmi et al. [17] proposed a weighted low-power routing protocol. The protocol selected the maximum weighted and minimum connected control 
set of network nodes based on the weights, which consisted of link stability, mobility and energy. Simulation results showed that the protocol was superior to other protocols in packet delivery rate, control message overhead, transmission delay and energy consumption. Nivedhitha et al. [18] proposed a dynamic multi-hop energy efficient routing protocol (DMEERP) aim to balance the path reliability ratio and energy consumption in ECNs. Simulation resulted that the novel protocol performed better in terms of packet delivery ratio, network lifetime, data flow, energy consumption, path reliability ratio, control overhead and delay, etc. Vipin bondre et al. [19], Ramakrishnan et al. [20] analyzed the performance of AODV, DSR, DSDV and other traditional routing protocols in emergency scenarios. However, they did not consider the mobility model of rescuers, nor the RUDs in disaster areas.

In this paper, we first propose a seismic intensity-based four-quadrant rescue mobility model (FQMM), and then, we simulate earthquake scene and propose a FQMM-based protocol for ECN. The main contributions of this paper are as follows.

Firstly, this paper gives the topology of ECN for the scene of post-earthquake emergency rescue. In the proposed post-earthquake ECN, both fixed nodes (e.g., emergency communication vehicles, base stations) and mobile nodes (e.g., Unmanned Aerial Vehicles, portable communication devices) are included. The characteristics of ECN are frequent link breakage, inconsistencies in data rates, incompatibility of resources, the temporary unavailability of needed resources and communications links.

Secondly, the FQMM mobility model for post-earthquake emergency rescue crews is proposed, which based on the topological structure of ECN. With FQMM, first responders not only can carry out grid search in disaster areas to ensure the effectiveness of rescue without missing any victims, but also can carry out radiation diffusion rescue from epicenter to ensure the worst affected areas are on top of the rescue list.

Finally, this paper proposed a FQMM-based protocol (FQMMBP) for ECN. According to the location of the mobile node, FQMMBP predicts hop counts from source to destination, so that it can update the routing table in real time by selecting the optimal nexthop node, thus improving package delivery rate, reducing end-to-end delay.

Although the routing protocol proposed in this paper only considers the factor of seismic intensity, the designed mobility model is universal and can be applied to other natural disasters, such as floods, fires, typhoons and so on.

\subsection{Problem description}

This paper aims to solve the problems of mobility, communication and rescue task arrangement in the earthquake rescue scenario. The detailed descriptions are as follows:

1. Mobility problem: Based on the common sense of earthquake, the core, rather than the rim of earthquake, has more important emergency to rescue. Therefore, we intend to solve the mobility problem that realizes more rescuers reach the core of earthquake with the minimal traveling time.

2. Rescue task arrangement problem: When the rescuers' mobility is finished, the paper will solve the problem of rescue task arrangement problem. There is more than one rescuers in each divided zone. And what is the optimal number of arranged rescuers for each zones should be modeled and solved via algorithms. 
3. Communication problem: Due to the mobility of rescuers, the paper should consider the real-time connectivity communication among the rescue vehicle and rescuers. The proposed routing protocols should guarantee the communication link from rescuers to rescue vehicles during the process of mobility and rescue task, which based on relay connective probability.

Overall, this paper mainly solves the problems defined as above.

\section{Method}

\subsection{Scenario and assumption}

In the post-earthquake ECN, the emergency communication vehicle (ECV) and base station (BS) are regarded as fixed nodes, while rescue crews equipped with individual communication equipment are regarded as mobile nodes. Unmanned aerial vehicle (UAV) can be used not only as a fixed node for relaying communication, but also as a mobile node for collecting disaster information. A typical post-earthquake ECN is shown in Fig. 1.

After the earthquake, rescuers equipped with individual communication equipment were scattered to various disaster areas for rescue. Each communication node in ECN, such as ECV, UAV and portable individual equipment (PIE), has its own communication coverage. Since ECV cannot reach the center of the disaster area due to traffic control, it starts at the midpoint of length, where rescue crews start and stop rescue missions, as shown in Fig. 1. In the rescue process, ECV can provide real-time communication for rescue crews. Due to its mobility and air superiority, UAV can go deep into the disaster area and play the role of communication relay or disaster information collector. However, due to the limited battery capacity and high costs of UAV, we are unable to deploy a large number of UAVs in the disaster area. Rescuers

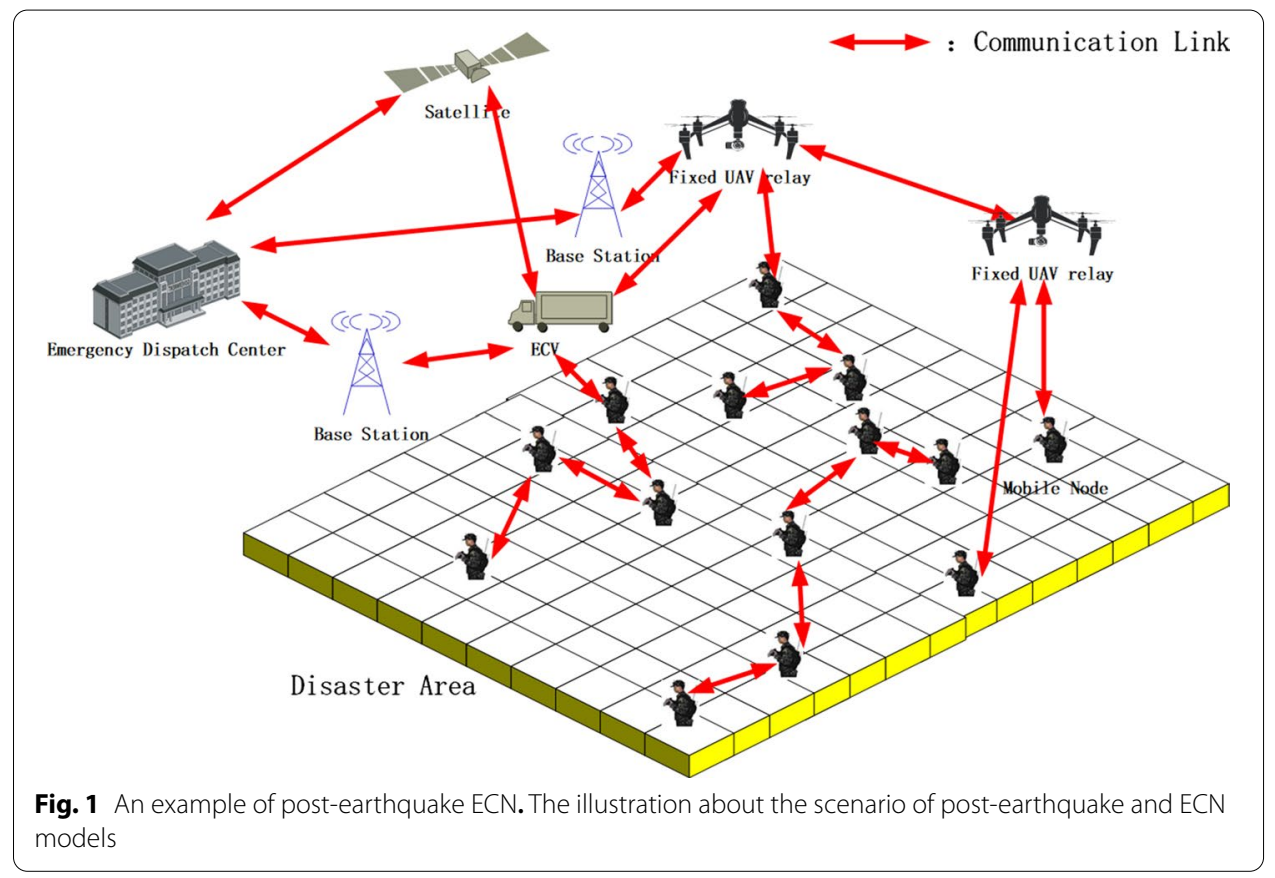


can enter the disaster area with PIE, however, due to the limited coverage, high cost and large energy consumption, PIE cannot play the role of hot spot or relay for a long time. Therefore, how to cooperate with the communication equipment in ECN to improve the communication efficiency is one of the key issues that need to be paid attention to in post-earthquake emergency rescue. Therefore, heterogeneous communication devices in ECN need to cooperate with each other through appropriate routing protocols to improve communication efficiency.

After the earthquake, we use rectangle to represent the earthquake influence range. The length and width of the rectangle are $a_{i}$ and $b_{i}(i=1 \ldots n)$, respectively. Before studying the mobile model, we propose the following assumptions for simulation calculation:

Assumption 1 In regions with equal seismic intensity, the values of RUD are equal.

Assumption 2 Each rescuer has got equal rescue efficiency.

Assumption 3 Rescue tasks in low RUD areas are at high priority. Rescue crews cannot start rescue tasks in higher RUD areas until rescue tasks in low RUD areas are completed.

Assumption 4 Random waypoint mobility model [21] for rescuers is used in each disaster area. In order to ensure the fairness and rationality of the rescue sequence, the rescuers are approximately evenly distributed in disaster areas with equal RUD values.

Assumption 5 Locations and number of rescue tasks are known by rescue crews in advance, since the Global Positioning System (GPS) is used.

Based on seismic intensity and number of rescuers in disaster areas, this paper proposes a four-quadrant mobility model for rescue crews, which improves the rescue efficiency and ensures the safety of the victims' lives and properties. The proposed mobility model mainly includes two aspects: Firstly, the most seriously affected areas are rescued as soon as possible; secondly, the rescue crews are allocated to each appropriate quadrant, which improves the rescue efficiency and shortens the time of rescue.

Our proposed protocols and algorithms are deployed into the rescuers, ECV and UAVs, which use the WSN technologies as platform and infrastructures. Considering the damage of base station and internet on the earthquake scenario, we use ad hoc networks to realize our proposed communication. The ECV is regarded as a tiny server, which is stopping at the rim of the damage area and calculating the RUD and mobility results for each rescuers. And all the rescuers' mobile information and rescue task status will be send back to the ECV. We assume that every rescuer is implemented an on-broad unit (OBU), which uses WSN technologies. The rescuers can share their basic information, including the real-time position, the task procedure, the remaining energy and the mobile speed, etc. The OBU can realize the rescuers' communication to other rescuers, ECV and UAVs. As the temporal relay, UAV will improve the communication performance when one of rescuer on the communication link is broken or exhaust energy. UAV will relay the broken communication link and re-arrange the task that exhausted rescuer to other working rescuers. 
Based on the overall idea, our proposed algorithms can be implemented by current technologies and can realize effective and real-time communication among rescuers, ECV, and UAVs.

\subsection{Disaster area division}

Disaster area is divided into $n$ layers according to seismic intensity. The epicenter is located in the first layer (RUD $=1$ ) where length is $a_{1}$ and width is $b_{1}$. We define RUD of each region as $1,2, \ldots, n$. Epicenter located at the center of rectangle where RUD $=1$. All areas are rectangular rings, except for the one with $\mathrm{RUD}=1$, which is a rectangle. ECV parks at the midpoint of the length outside the rectangular ring with RUD $=n$, as shown in Fig. 2.

The length and width of each disaster area are marked as $\left\{\operatorname{RUD} \mid a_{i}, b_{i}\right\}$; then, each region can be expressed as follows:

$$
\begin{aligned}
& \text { Region 1: }\left\{\operatorname{RUD}=1 \mid a_{1}, b_{1}\right\} ; \\
& \text { Region 2: }\left\{\text { RUD }=2 \mid a_{2}=a_{1}+\frac{a_{1}}{2}+\frac{a_{1}}{2}=2 a_{1}, b_{2}=b_{1}+\frac{b_{1}}{2}+\frac{b_{1}}{2}=2 b_{1}\right\} ; \\
& \text { Region 3: }\left\{\operatorname{RUD}=3 \mid a_{3}=a_{2}+\frac{a_{2}}{2}+\frac{a_{2}}{2}=2 a_{2}=4 a_{1}, b_{3}=b_{2}+\frac{b_{2}}{2}+\frac{b_{2}}{2}=2 b_{2}=4 b_{1}\right\} ;
\end{aligned}
$$

According to mathematical induction, we can get:

Region $i$ : $\left\{\mathrm{RUD}=3 \mid a_{i}=2 a_{i-1}=2^{i-1} a_{1}, b_{i}=2 b_{i-1}=2^{i-1} b_{1}\right\}$.

\subsection{FQMM model}

In this section, a four-quadrant rescuers allocation scheme is proposed. Based on this scheme, a four-quadrant mobility model (FQMM) for rescuers is proposed. The objectives of the allocation scheme are: (1) carrying out grid search in disaster areas to ensure the effectiveness of rescue without missing any victims; (2) carrying out radiation diffusion rescue from epicenter to ensure the worst affected areas are on top of the rescue list. From the disaster area division discussed in Sect. 3.2, it can be seen that when

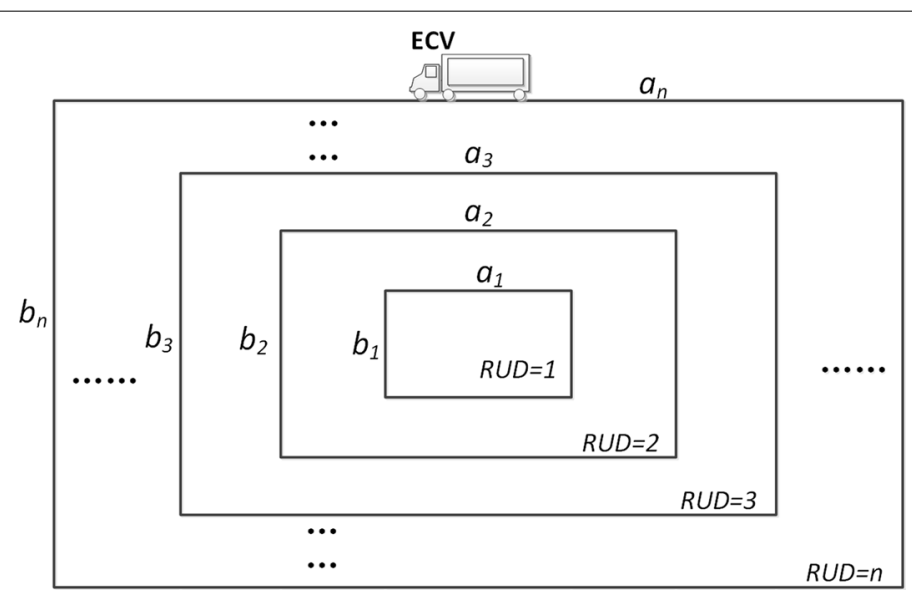

Fig. 2 Disaster area division according to RUD. The illustration about the main idea of disaster area division, which means that the core of earthquake will be divided first that its RUD denotes 1 
$R U D=1$, the region is a rectangle, and when $R U D>1$, the regions are rectangular rings. Therefore, the discussion of rescuers allocation scheme includes two aspects: RUD $=1$ and RUD $>1$. The core ideas of rescuers allocation scheme for regions with RUD $>1$ are similar, though the lengths of the regions are different.

\subsubsection{Mobility model for rescuers in regions with $R U D=1$}

Five steps (Step 1 to Step 5) are included in the mobility model of rescuers for regions with RUD $=1$.

Step 1 As shown in Fig. 3, we establish a coordinate system which takes the epicenter as the origin. $X$ and $Y$ axes are the length and width of the rectangle. Four quadrants, $\mathrm{QID}=1, \mathrm{QID}=2, \mathrm{QID}=3$ and $\mathrm{QID}=4$ are counter-clockwise defined according to the definition of coordinate system. Each quadrant is described as follows:

Quadrant 1: $\{$ RUD $=1 \mid \mathrm{QID}=1\} ;$ Quadrant 2: $\{\mathrm{RUD}=1 \mid \mathrm{QID}=2\}$;

Quadrant 3: $\{$ RUD $=1 \mid \mathrm{QID}=3\} ;$ Quadrant 4: $\{\mathrm{RUD}=1 \mid \mathrm{QID}=4\}$.

Suppose that the total number of rescuers is $M$, and the total number of rescue tasks in the area with $\mathrm{RUD}=1$ is $Q$, which is, $\mathrm{RT}_{\mathrm{QL}=0}^{\mathrm{RUD}=1}=Q$, where $\mathrm{RT}_{\mathrm{QL}=0}^{\mathrm{RUD}=1}$ stands for total number of rescue tasks to be completed in disaster areas with RUD $=1$ and $\mathrm{QL}=0$. The number of tasks to be performed in each quadrant is described as: $\mathrm{RT}_{\mathrm{QID}=k}^{\mathrm{RUD}=1}=Q / 4, \quad(k=1,2,3,4)$, where $\mathrm{RT}_{\mathrm{QID}=k}^{\mathrm{RUD}=1}$ stands for the number of tasks to be performed in disaster areas with $\mathrm{RUD}=1$ and $\mathrm{QID}=k(k=1,2,3,4)$. Rescuers are evenly allocated to four quadrants with $\mathrm{QL}=1$, and the number of rescuers allocated (NRA) in each quadrant can be discussed from four cases (Case 1 to Case 4) as follows:

Case 1: $\operatorname{Mod}[M / 4]=0$, ere function $\operatorname{Mod}[x / y]$ is used to return the remainder of the division of two numbers $x, y$. In this case, a quarter of rescuers are evenly allocated to four quadrants with $\mathrm{QL}=1$, which is:

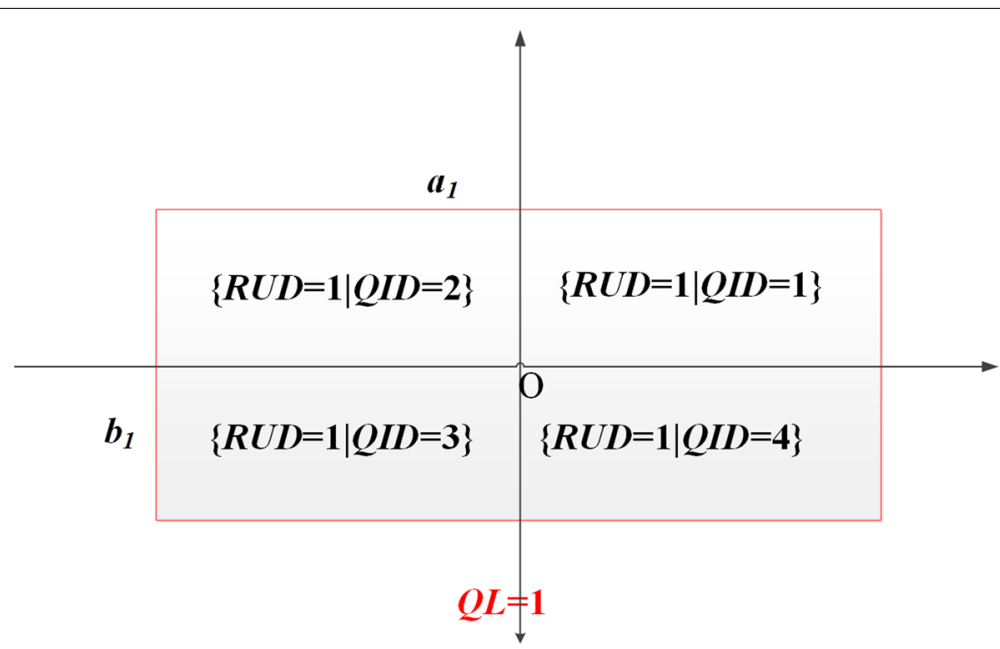

Fig. 3 Disaster area with $\mathrm{RUD}=1$ and $\mathrm{QL}=1$. The illustration about the four quadrants division idea in $R \cup D=1$ for the first time 


$$
\mathrm{NRA}_{\mathrm{QID}=k}^{\mathrm{RUD}=1}=\frac{M}{4}, \quad(k=1,2,3,4)
$$

where $\mathrm{NRA}_{\mathrm{QID}=k}^{\mathrm{RUD}=1}$ stands for the number of rescuers allocated in each quadrant with $\mathrm{RUD}=1$ and $\mathrm{QID}=k$.

Case 2: $\operatorname{Mod}[M / 4]=1$. In this case, one more rescuer is allocated to Quadrant 1, which are:

$$
\mathrm{NRA}_{\mathrm{QID}=1}^{\mathrm{RUD}=1}=\frac{M-1}{4}+1
$$

where $\mathrm{NRA}_{\mathrm{QID}=1}^{\mathrm{RUD}=1}$ stands for the number of rescuers allocated in Quadrant 1 with $\mathrm{RUD}=1$, and $\mathrm{NRA}_{\mathrm{QID} \neq 1}^{\mathrm{RUD}=1}$ stands for the number of rescuers allocated in Quadrant 2, Quadrant 3 and Quadrant 4 with RUD $=1$.

Case 3: $\operatorname{Mod}[M / 4]=2$. In this case, one more rescuer is allocated to Quadrant 1 and Quadrant 2, respectively, which are:

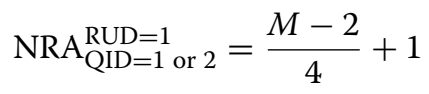

where $\mathrm{NRA}_{\mathrm{QID}=1}^{\mathrm{RUD}=1} 2$ stands for the number of rescuers allocated in Quadrant 1 and Quadrant 2 with $\mathrm{RUD}=1$, and $\mathrm{NRA}_{\mathrm{QID}=3 \text { or } 4}^{\mathrm{RUD}=1}$ stands for the number of rescuers allocated in Quadrant and Quadrant 4 with RUD $=1$.

Case 4: $\operatorname{Mod}[M / 4]=3$. In this case, one more rescuer is allocated to Quadrant 1 , Quadrant 2, Quadrant 4 which are:

$$
\mathrm{NRA}_{\mathrm{QID} \neq 3}^{\mathrm{RUD}=1}=\frac{M-3}{4}+1
$$

where $\mathrm{NDN}_{\mathrm{QID} \neq 3}^{\mathrm{RUD}=1}$ stands for the number of rescuers allocated in Quadrant 1, Quadrant 2 and Quadrant 4 with $\mathrm{RUD}=1$, and $\mathrm{NRA}_{\mathrm{QID}=3}^{\mathrm{RUD}=1}$ stands for the number of rescuers allocated in Quadran3 with RUD $=1$.

Step 2 According to the procedure of step 1, each quadrant with RUD $=1$ is further divided into four secondary quadrants as shown in Fig. 4. Two elements are included in QID set, which record the quadrant ID from the first level to the last level. For example, $\{\mathrm{RUD}=1 \mid \mathrm{QID}=\{1,3\}\}$ in Fig. 4 means region with $\mathrm{QL}=1$ is located in Quadrant 1 and region with $\mathrm{QL}=2$ is located in Quadrant 3.

Step 3 For $\mathrm{QL}=x$, replace the value of $M$ with $\mathrm{RT} \mathrm{QL}=x$, repeat Step 1 and calculate the number of rescuers allocated for each quadrant with $\mathrm{QL}=x$.

Step 4 Repeat Step 2 and Step 3, until any of the following Abort conditions is satisfied.

Abort condition 1 There is only one rescuer in each area of quadrant with $\mathrm{QL}=x$, which is: $\mathrm{NRA}_{\mathrm{QID}=k}^{\mathrm{RUD}=1}=1, \quad(k=1,2,3,4)$, where $\mathrm{NRA}_{\mathrm{QID}=k}^{\mathrm{RUD}=1}$ is the number of rescuers allocated in Quadrant $k(k=1,2,3,4)$ with $\mathrm{RUD}=1$ and $\mathrm{QL}=x$. Under this condition, further quadrant division is no longer meaningful, so the single rescuer will complete all rescue tasks in the region of quadrant with $\mathrm{QL}=x$.

Abort condition 2 The number of rescue tasks is less than that of rescuers assigned in the quadrant with $\mathrm{QL}=x$, which is: $\mathrm{NRA}_{\mathrm{QID}=k}^{\mathrm{RUD}=1} \geq \mathrm{RT}_{\mathrm{QID}=k}^{\mathrm{RUD}=1}, \quad(k=1,2,3,4)$, where $\mathrm{NRA}_{\mathrm{QID}=k}^{\mathrm{RUD}=1}$ is the number of rescuers allocated in Quadrant $k(k=1,2,3,4)$ with $\mathrm{RUD}=1$ and $\mathrm{QL}=x$, and $\mathrm{RT}_{\mathrm{QID}=k}^{\mathrm{RUD}=1}$ is number of rescue tasks in Quadrant $k(k=1,2,3$, 


\begin{tabular}{|c|c|c|c|c|c|}
\hline & & & $a_{1}$ & & \\
\hline & $\{R U D=1 \mid Q I D=\{2,2\}\}$ & $\{R U D=1 \mid Q I D=\{2,1\}\}$ & $\{R U D=1 \mid Q I D=\{1,2\}\}$ & $\{R U D=1 \mid Q I D=\{1,1\}\}$ & \\
\hline \multirow[t]{4}{*}{$b_{1}$} & $\{R U D=1 \mid Q I D=\{2,3\}\}$ & $\{R U D=1 \mid Q I D=\{2,4\}\}$ & $\{R U D=1 \mid Q I D=\{1,3\}\}$ & $\{R U D=1 \mid Q I D=\{1,4\}\}$ & \\
\hline & $\{R U D=1 \mid Q I D=\{3,2\}\}$ & $\{R U D=1 \mid Q I D=\{3,1\}\}$ & ${ }^{0}\{R U D=1 \mid Q I D=\{4,2\}\}$ & $\{R U D=1 \mid Q I D=\{4,1\}\}$ & \\
\hline & $\{R U D=1 \mid Q I D=\{3,3\}\}$ & $\{R U D=1 \mid Q I D=\{3,4\}\}$ & $\{R U D=1 \mid Q I D=\{4,3\}\}$ & $\{R U D=1 \mid Q I D=\{4,4\}\}$ & \\
\hline & & \multicolumn{2}{|c|}{$Q L=2$} & & \\
\hline \multicolumn{6}{|c|}{$\begin{array}{l}\text { Fig. } 4 \text { Disaster area with } \mathrm{RUD}=1 \text { and } \mathrm{QL}=2 \text {. Based on Fig. 3, this figure divides the area further from } 4 \text { to } 16 \\
\text { quadrants }\end{array}$} \\
\hline
\end{tabular}

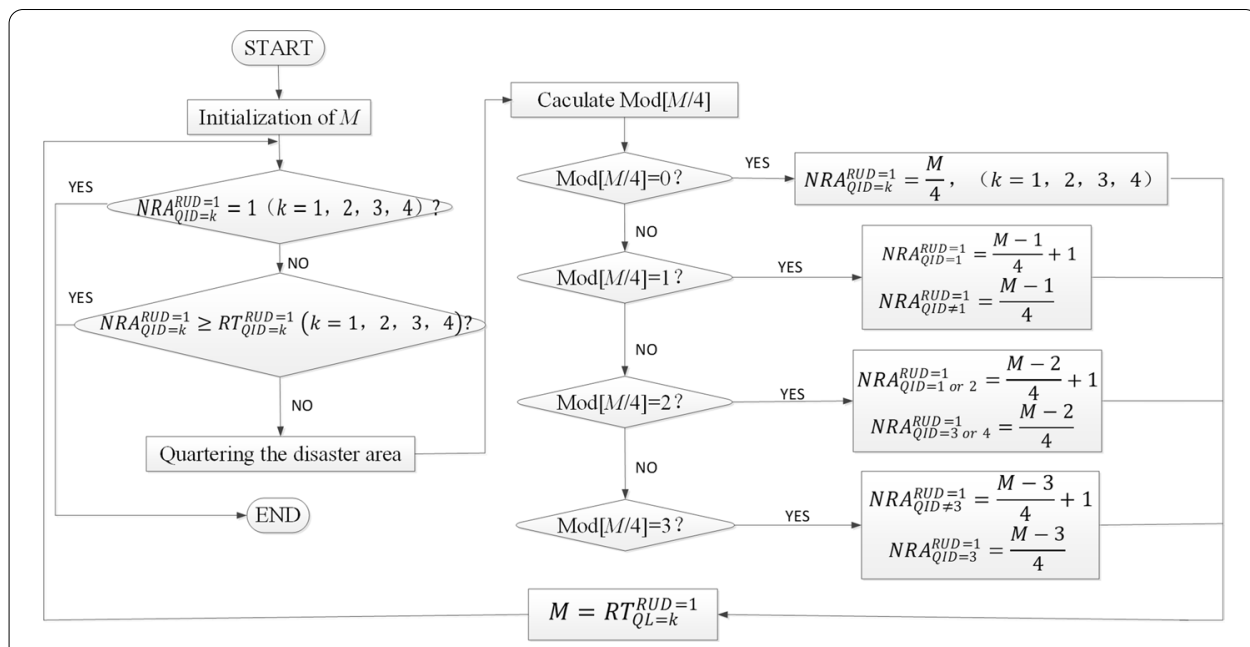

Fig. 5 Allocation process of rescue nodes in disaster area with $R U D=1$. The flowchart to describe the entire process of rescue nodes mobility in the RUD $=1$ area

4) with $\mathrm{RUD}=1$ and $\mathrm{QL}=x$. Under this condition, each rescuer is assigned to at most one rescue task, and there are unoccupied rescuers in the area.

Step 5 Quadrant division is suspended and rescuers begin to perform their own rescue tasks.

Figure 5 shows the algorithm flow of rescue node allocation in rectangular disaster area when $\mathrm{RUD}=1$.

\subsubsection{Mobility model for rescuers in regions with RUD $>1$}

According to the mobility model, rescue crews cannot start rescue tasks in higher RUD areas until rescue tasks in low RUD areas are completed. As shown in Fig. 6, in the gray area with $\mathrm{RUD}=1$, rescuers are carrying out rescue tasks. When the task is over, rescuers will be assigned to the white rectangular ring area with RUD $=2$. 

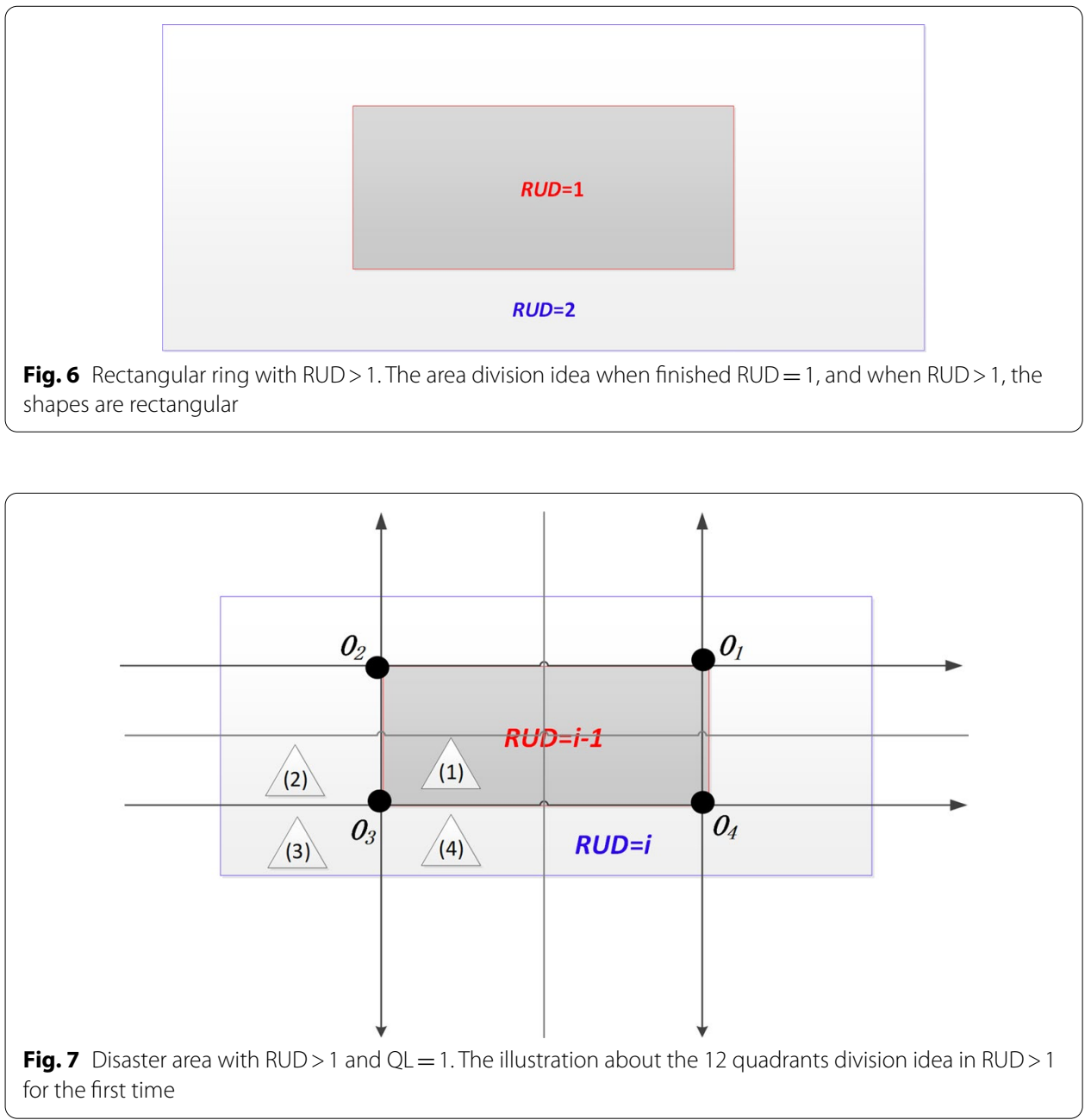

Five steps (Step 6 to Step 10) are included in the mobility model of rescuers for regions with RUD $>1$.

Step 6 As shown in Fig. 7, we establish four coordinate systems which takes $O_{1}, O_{2}$, $\mathrm{O}_{3}$ and $\mathrm{O}_{4}$ as the origins, respectively. Then, the rectangular ring is equally divided into twelve quadrants (the white parts in Fig. 7) by four coordinate systems. In each coordinate system, four quadrants are equal with each other in area, as shown in Fig. 7 where four triangle marks.

Step 7 It can be seen from Step 6 that in each coordinate system, there are three quadrants waiting for allocation of rescuers, and the left quadrant is allocated with rescue crews. As shown in Fig. 7 , in the coordinate system with $\mathrm{O}_{3}$ as origin, there are four quadrants where Triangle (1), Triangle (2), Triangle (3) and Triangle (4) marks. Area where Triangle (1) marks is allocated with rescue crews, while areas where Triangle (2), Triangle (3) and Triangle (4) marks are waiting for rescuers allocations. We take coordinate system $O_{3}$ as an example. Suppose there are $M$ rescuers in Quadrant 1 with RUD $=i-1$, which marked with Triangle (1). There are $Q$ rescue tasks in areas with $\mathrm{RUD}=i$, which is: $\mathrm{RT}_{\mathrm{QL}=0}^{\mathrm{RUD}=i}=Q$. Rescuers are evenly allocated to three quadrants [Triangle (2), Triangle (3) and Triangle (4)] with $\mathrm{QL}=1$, and the number of 
rescuers allocated (NRA) in each quadrant can be discussed from three cases (Case 5 to Case 7) as follows:

Case $5 \operatorname{Mod}[M / 3]=0$, where function $\operatorname{Mod}[x / y]$ is used to return the remainder of the division of two numbers $x, y$. In this case, one-third rescuers are evenly allocated to three quadrants, which is:

$$
\mathrm{NRA}_{\mathrm{QID}=k}^{\mathrm{RUD}=i}=\frac{M}{3}, \quad(k=2,3,4)
$$

where $\mathrm{NRA}_{\mathrm{QID}=k}^{\mathrm{RUD}=i}$ stands for the number of rescuers allocated in each quadrant with $\mathrm{RUD}=i$ and $\mathrm{QID}=k$.

Case $6 \operatorname{Mod}[M / 3]=1$. In this case, one more rescuer is allocated to Quadrant 2, which are:

$$
\mathrm{NRA}_{\mathrm{QID}=2}^{\mathrm{RUD}=i}=\frac{M-1}{3}+1
$$

where $\mathrm{NRA}_{\mathrm{QID}=2}^{\mathrm{RUD}=i}$ stands for the number of rescuers allocated in Quadrant 2 with $\mathrm{RUD}=i$, and $\mathrm{NRA} \mathrm{A}_{\mathrm{QID}=3 \text { or } 4}^{\mathrm{RUD}}$ stands for the number of rescuers allocated in Quadrant 3 and Quadrant 4 with $R=i$.

Case $7 \operatorname{Mod}[M / 3]=2$. In this case, one more rescuer is allocated to Quadrant 2 and Quadrant 4, respectively, which are:

$$
\mathrm{NRA}_{\mathrm{QID}=2 \text { or } 4}^{\mathrm{RUD}=i}=\frac{M-2}{3}+1
$$

where $\mathrm{NRA} \mathrm{QID}_{\mathrm{QID}}^{\mathrm{RUD}=2}$ or 4 stands for number of rescuers allocated in Quadrant 2 and Quadrant 4 with $\mathrm{RUD}=i$, and $\mathrm{NRA}_{\mathrm{QID}=3}^{\mathrm{RUD}=i}$ stands for the number of rescuers allocated in Quadrant 3 with $\mathrm{RUD}=i$.

The number of rescue tasks in each quadrant with $R U D=i$ is: $\mathrm{RT}_{\mathrm{QID}=k}^{\mathrm{RUD}=i}=Q / 12, \quad(k=2,3,4)$, where $\mathrm{RT}_{\mathrm{QID}=k}^{\mathrm{RUD}=i}$ stands for the number of rescue tasks in Quadrant $k(k=2,3,4)$ wh $\mathrm{RUD}=i$.

Step 8 Quadrants $(\mathrm{QL} \geq 2)$ division methods are the same as illustrated in Step 6, which take the middle points of quadrants with $\mathrm{QL}=1$ as the origins and establish the coordinate systems. Repeat Step 7, until any of the following Abort conditions is satisfied.

Abort condition 3 There is only one rescuer in each area of quadrant with $\mathrm{QL}=x$, which is: $\mathrm{NRA}_{\mathrm{QID}=k}^{\mathrm{RUD}=i}=1, \quad(k=1,2,3,4)$, where $\mathrm{NRA}_{\mathrm{QID}=k}^{\mathrm{RUD}=i}$ is the number of rescuers allocated in Quadrant $k(k=1,2,3,4)$ with $\mathrm{RUD}=i$ and $\mathrm{QL}=x$. Under this condition, further quadrant division is no longer meaningful, so the single rescuer will complete all rescue tasks in the region of quadrant with $\mathrm{QL}=x$.

Abort condition 4 The number of rescue tasks is less than that of rescuers assigned in the quadrant with $\mathrm{QL}=x$, which is: $\mathrm{NRA}_{\mathrm{QID}=k}^{\mathrm{RUD}=i} \geq \mathrm{RT}_{\mathrm{QID}=k}^{\mathrm{RUD}=i}, \quad(k=1,2,3,4)$, where $\mathrm{NRA}_{\mathrm{QID}=k}^{\mathrm{RUD}=i}$ is the number of rescuers allocated in Quadrant $k(k=1,2,3,4)$ with $\mathrm{RUD}=i$ and $\mathrm{QL}=x$, and $\mathrm{RT} \mathrm{QID}=k$ is number of rescue tasks in Quadrant $k(k=1,2,3,4)$ with $\mathrm{RUD}=i$ and $\mathrm{QL}=x$. Under this condition, each rescuer is assigned to at most one rescue task, and there are unoccupied rescuers in the area.

Step 9 Quadrant division is suspended and rescuers begin to perform their own rescue tasks. 
Figure 8 shows the algorithm flow of rescue node allocation in rectangular disaster area when RUD $>1$.

\subsection{FDMM-based routing protocol}

In this section, a proposal of FQMM-based protocol (FQMMBP) for ECN is presented. First, we discuss route discovery and maintenance of FQMMBP, and then we propose FQMMBP protocol by analyzing the communication mechanism of nodes in ECN.

Post-earthquake ECN, which is a hybrid self-organized network, mainly includes ECV, UAV, portable devices carried by rescue crews, base station, satellite, etc. Those communication devices can be classified as fixed communication nodes (such as ECV, base station, UAV used for relay.) and mobile communication nodes (such as portable devices carried by rescue crews, satellite.). As shown in Fig. 1, each portable device is equipped with a wireless communication module. Rescuers can use portable devices to build self-organized networks, through which they can communicate with each other and complete the transmission process of data packets, such as receiving, transmitting and relaying. Rescuers can also communicate with ECV, UAV and other fixed nodes within the coverages of portable devices. As a fixed communication node, ECV can communicate with rescuers and UAVs in the disaster area through downlink, or with the base station, satellite and other communication facilities through uplink. Through the backbone network, commanders can get the information about rescue crews, rescue process and suffering condition in time, so as to make scientific rescue decision and emergency response quickly.

We define the communication radius of mobile node as $R$. The mobile node sends data to the emergency communication vehicle in the way of multi-hop. In such communication mode, the source is the mobile node in the disaster area, the destination is ECV at the edge of the disaster area, and the relay nodes are mobile nodes or fixed nodes (UAVs, etc.) in the disaster area. On the premise of ensuring the stability of communication links, we choose relay nodes or links that can minimize the delay for data transmission. Based on the mobility model we discussed in Sect. 3.3, it can be seen that rescue crews are nearly evenly distributed in disaster areas with the same RUD. In order to ensure the

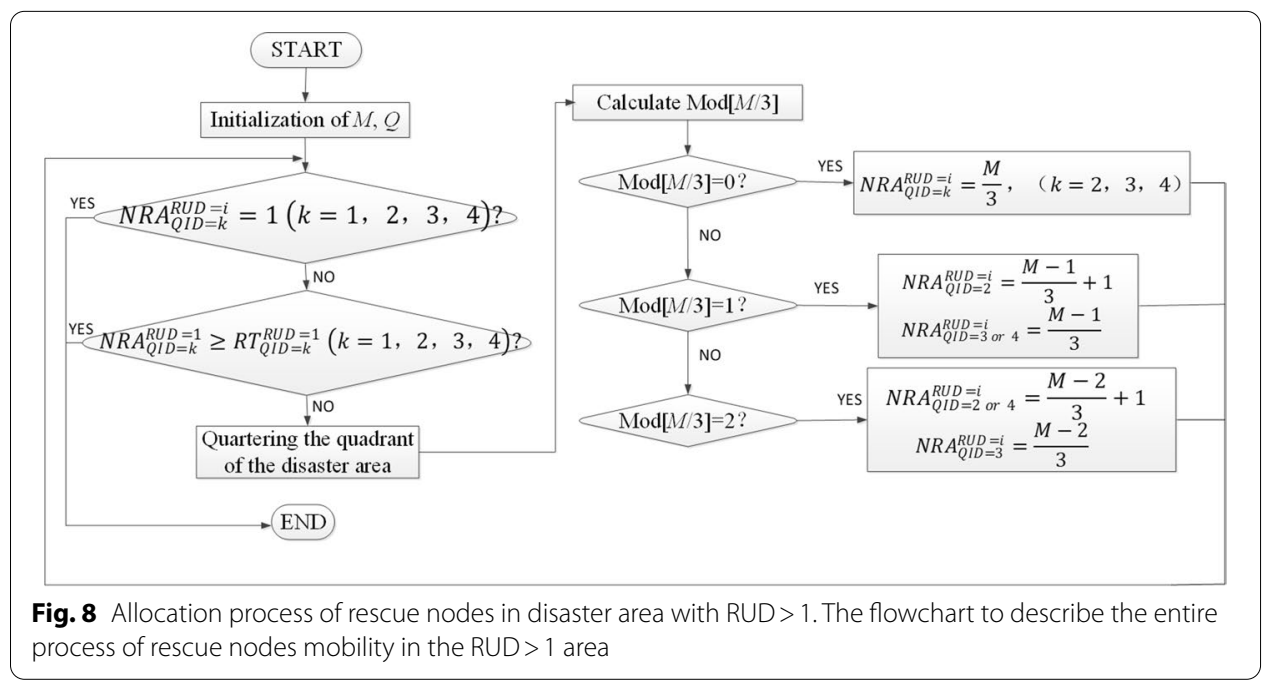


stability of the communication link in the rescue process, we place fixed UAVs at the edges of disaster areas with different RUD values; however, they do not undertake the rescue tasks. When all rescue tasks in the disaster area with a specific RUD value are completed, the relay UAV in the area returns to the location of ECV.

\subsubsection{Routing setup}

In order to reduce the data transmission delay, we select the next hop relay node according to RUD value, QL and QID of each region where mobile nodes are located in. RUD and QL are used to define the level of the region where mobile nodes are located in. QID is a set which includes 1,2, 3 and 4, and there are QL elements in the set. According to the set, the mobile nodes learn the division of the regions and the quadrants position, so as to select the appropriate next hop mobile node. Therefore, in the process of routing discovery, it is necessary to extend the routing request (RREQ) message, so as to meet the requirements of our proposed communication mechanism of FQMMBP. We improved the traditional RREQ message by adding four new fields: RUD, QL, QID and QW. RUD describes the area where nodes are located and judge whether the area is a rectangle or a rectangular ring as shown in Fig. 2. QL describes the level of the quadrant where nodes are located, and it determines the relative position of the mobile rescue node. QID describes the division of the quadrant where nodes are located and judge its relative position to ECV. When the QL of quadrants are the same, QID helps pick up the mobile rescue node which is closer to ECV. QW calculates weights of nodes according to QL and QID, so as to select the most appropriate next-hop relay node.

In the process of route discovery, nodes broadcast RREQ messages to send request information to their neighbors. The improved RREQ data format is shown in Table 2.

Each field of RREQ data format is described as follows:

Type: The length of this field is 8bit, and the type value of RREQ is 1.

Type: The length of this field is 8bit, and the type value of RREQ is 1.

Flags: The length of this field is 5 bits, and five identities (J, R, G, D, and U) are included in this field. "J" is a joint flag and is generally used for multicast. " $R$ " is for route repairing and is used for multicast transmission. "G" represents the list of nodes around ECV which can be communicated. Flag "G" determines whether the RREQ message can be directly sent to the destination or not. " $\mathrm{D}$ " is the reply flag of the destination node which determines whether the destination node is allowed to reply to the received RREQ message or not. "U" is the flag of unknown serial number. $\mathrm{U}=1$ means that the serial number of the node is unknown.

Reserved: Reserved field for further improvement of RREQ message.

Hop Count: This field registers the hop counts that RREQ passes from the source node to the current node.

RREQ ID: This field is the unique identity of RREQ message.

RUD: Rescue Urgency Degree, which is inversely proportional to the seismic intensity value in this area.

QL: Quadrant Level, which is the number of times the quadrant divided.

QID: Quadrant ID. 


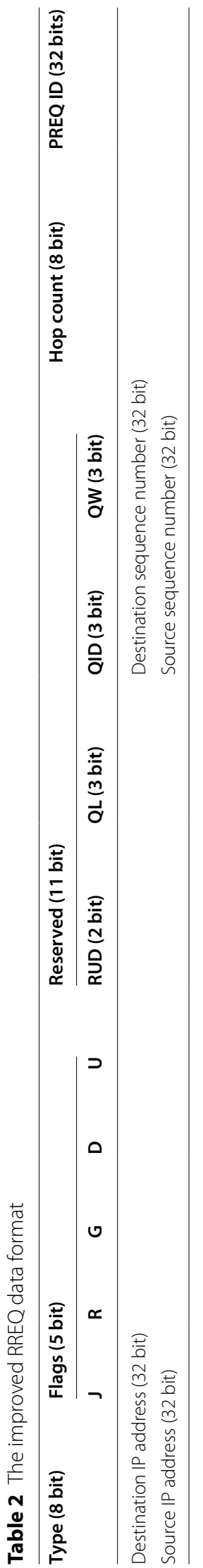


QW: Weights of nodes in quadrant.

\subsubsection{Communication mechanism}

As shown in Table 2, for fixed UAV nodes located in different RUD areas, all fields of RREQ message are empty except for "RUD." The "RUD" field describes the UAV's location. Suppose that there is one UAV relay node in each region with RUD $=i(i=1$ to $n)$, and the UAVs in two adjacent regions can communicate with each other directly. Then, there are $n+1$ fixed communication nodes in the disaster area ( $n$ fixed UAVs and one ECV). Mobile nodes in regions with $\operatorname{RUD}=i(i=1$ to $n)$ can transmit data to the fixed UAV node either by one hop or by multiple hops. After that, the data can be transmitted to ECV through multi-hop between fixed nodes without relying on other mobile nodes. In that case, we need to select the most appropriate next hop relay node (mobile node or fixed node) when the next hop candidate is in different quadrants. It can be seen from Fig. 2 that Quadrant 1 and Quadrant 2 are closer to ECV than Quadrant 3 and Quadrant 4, so the weights of Quadrant 1 and Quadrant 2 should be greater than that of Quadrant 3 and Quadrant 4. As shown in Fig. 9, the distance between the center of each quadrant and ECV is:

$$
\begin{aligned}
& D_{\mathrm{QID}}= \begin{cases}c & \mathrm{QID}=1 \text { or } 2 \\
d & \mathrm{QID}=3 \text { or } 4\end{cases} \\
& c=\sqrt{\left(\frac{a}{4}\right)^{2}+\left(\frac{b}{4}\right)^{2}} \\
& d=\sqrt{\left(\frac{a}{4}\right)^{2}+\left(\frac{3 b}{4}\right)^{2}}
\end{aligned}
$$

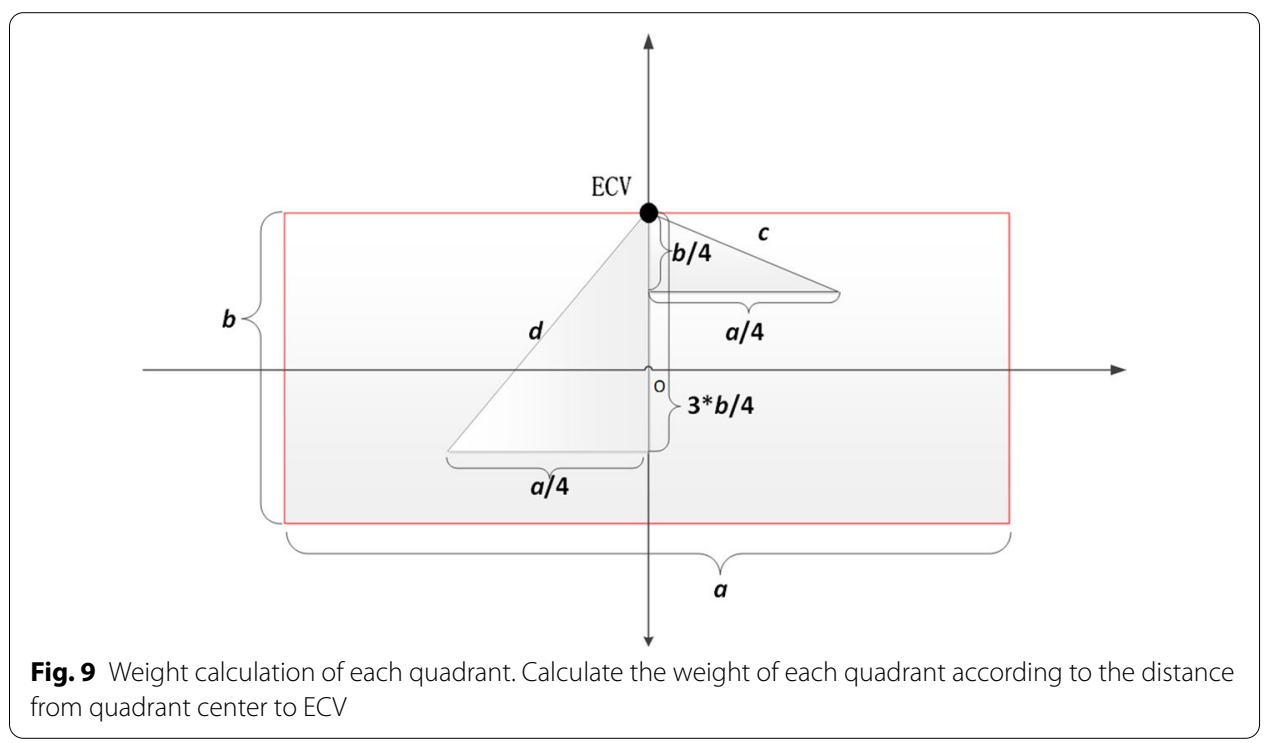


where $a, b$ are length and width of disaster area, respectively. $D_{P H}$ stands for the distance between the center of each quadrant and ECV. $c$ is the distance between the center of Quadrant 1 and ECV, which equals with the distance between the center of Quadrant 2 and ECV. $d$ is the distance between the center of Quadrant 3 and ECV, which equals with the distance between the center of Quadrant 4 and ECV. Take the weight of Quadrant 1 (or Quadrant 2) as the benchmark, which is: $W_{\mathrm{QID}=1 \text { or } 2}=1$, where $W_{\mathrm{QID}=1 \text { or } 2}$ is the weight of Quadrant 1 or Quadrant 2. The weight of Quadrant 3 (or Quadrant 4) can be expressed as:

$$
W_{\mathrm{QID}=3 \text { or } 4}=\frac{d}{c}=\frac{\sqrt{a^{2}+9 b^{2}}}{\sqrt{a^{2}+b^{2}}}
$$

where $W_{\mathrm{QID}=3}$ or 4 is the weight of Quadrant 3 or Quadrant 4.

Definition $\mathrm{QID}_{i}$ is the Quadrant ID with $\mathrm{QL}=i$. For example, $\mathrm{QID}_{3}=4$ means Quadrant 4 is with $\mathrm{QL}=3$. From Eqs. (1) and (4), we can get:

$$
D_{\mathrm{QID}_{\mathrm{i}}}= \begin{cases}c_{i} & \mathrm{QID}_{i}=1 \text { or } 2 \\ d_{i} & \mathrm{QID}_{i}=3 \text { or } 4\end{cases}
$$

where $D_{\mathrm{QID}_{i}}$ stands for the distance between the center of quadrant with $\mathrm{QID}_{i}$ and ECV. $c_{i}$ is distance between the center of Quadrant 1 with $\mathrm{QL}=i$ and $\mathrm{ECV}$, which equals with the distance between the center of Quadrant 2 with $\mathrm{QL}=i$ and ECV. $d_{i}$ is distance between the center of Quadrant 3 with $\mathrm{QL}=i$ and $\mathrm{ECV}$, which equals with the distance between the center of Quadrant 4 with $\mathrm{QL}=i$ and ECV. From Eq. (4), we can get:

$$
W_{\mathrm{QID}_{i}}=\frac{\sqrt{a_{i}^{2}+9 b_{i}^{2}}}{\sqrt{a_{i}^{2}+b_{i}^{2}}}
$$

where $W_{\mathrm{QID}_{i}}$ is the weight of node located in Quadrant $\mathrm{QID}_{i} . a_{i}, b_{i}$ are length and width of disaster area with $\mathrm{QL}=i$, respectively. According to the QID and QL, we can get the total weight of each node in quadrants with different levels by:

$$
\mathrm{QW}=\sum_{i=1}^{\mathrm{QL}} W_{\mathrm{QID}_{i}}
$$

where QW is the total weight of one node in quadrants with different levels. $W_{\mathrm{QID}_{i}}$ is the weight of node located in Quadrant $\mathrm{QID}_{i}$.

Suppose two nodes are located in Quadrant 1 and Quadrant 2, respectively, and the QW values of the two nodes are equal. According to FQMM, there is a higher probability that more rescuers are allocated in Quadrant 1 than in Quadrant 2. In that case, it is better to choose next hop nodes in Quadrant 1 than in Quadrant 2, because the probability of connecting to the destination node is higher. As a result, the priority of each quadrant is as follows: Quadrant 1>Quadrant 2> Quadrant 4> Quadrant 3. When the QW values of the nodes are equal, we can choose the node in the high priority quadrant as the next hop node. 


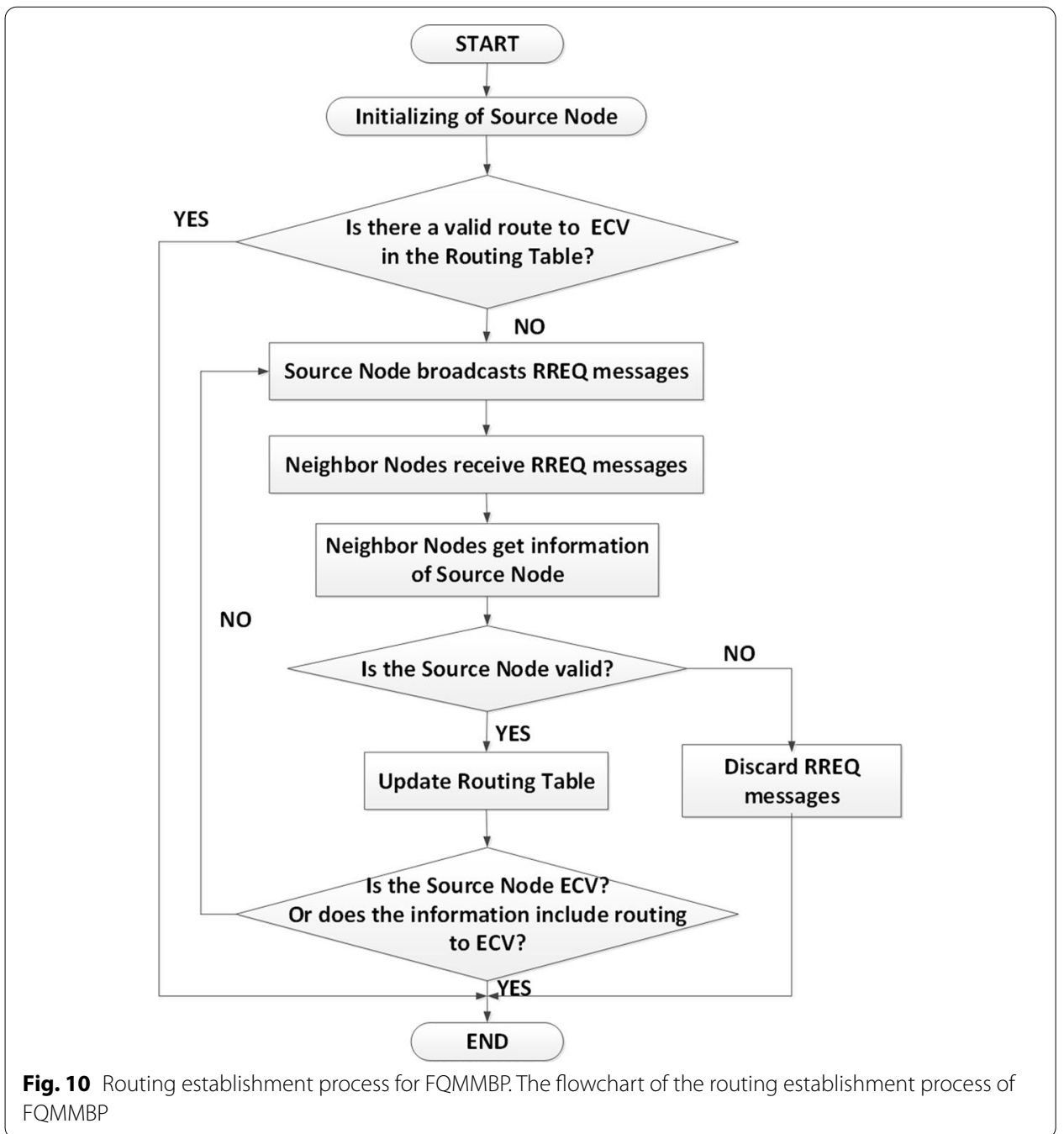

\subsubsection{Routing discover and maintain}

In this section, we propose FQMMBP protocol based on FQMM. Routing discovery and routing maintenance process are illustrated in Figs. 10 and 11, respectively.

As shown in Fig. 10, source node (denoted as $N_{S}$ ) checks whether there is a route to ECV. If there is no route to ECV, the node broadcasts RREQ message to its neighbor node (denoted as $N_{\mathrm{N}}$ ). After receiving the message, $N_{\mathrm{N}}$ first checks whether $N_{\mathrm{S}}$ is a valid node. If $N_{\mathrm{S}}$ is invalid, $N_{\mathrm{N}}$ discards the received RREQ message; otherwise, $N_{\mathrm{N}}$ updates the routing table and finds out whether there is routing information to ECV. If there is no route to ECV, $N_{\mathrm{N}}$ forwards the received RREQ message. Repeat the broadcasting and checking process until one or more valid routes to the ECV are found.

As shown in Fig. 11, source node (denoted as $N_{S}$ ) sends hello packets to the candidate nodes within its coverage by broadcasting and receives RREQ replies from them. $N_{\mathrm{S}}$ determines whether there is a RREQ reply sent by ECV by checking whether there is a candidate node with RUD $=n$ and both QL and QID are empty in RREQ reply. If there is an ECV within the coverage of $N_{S}$, data packets are directly transmitted to ECV which is the destination of the communication, and communication ends. If there is no 


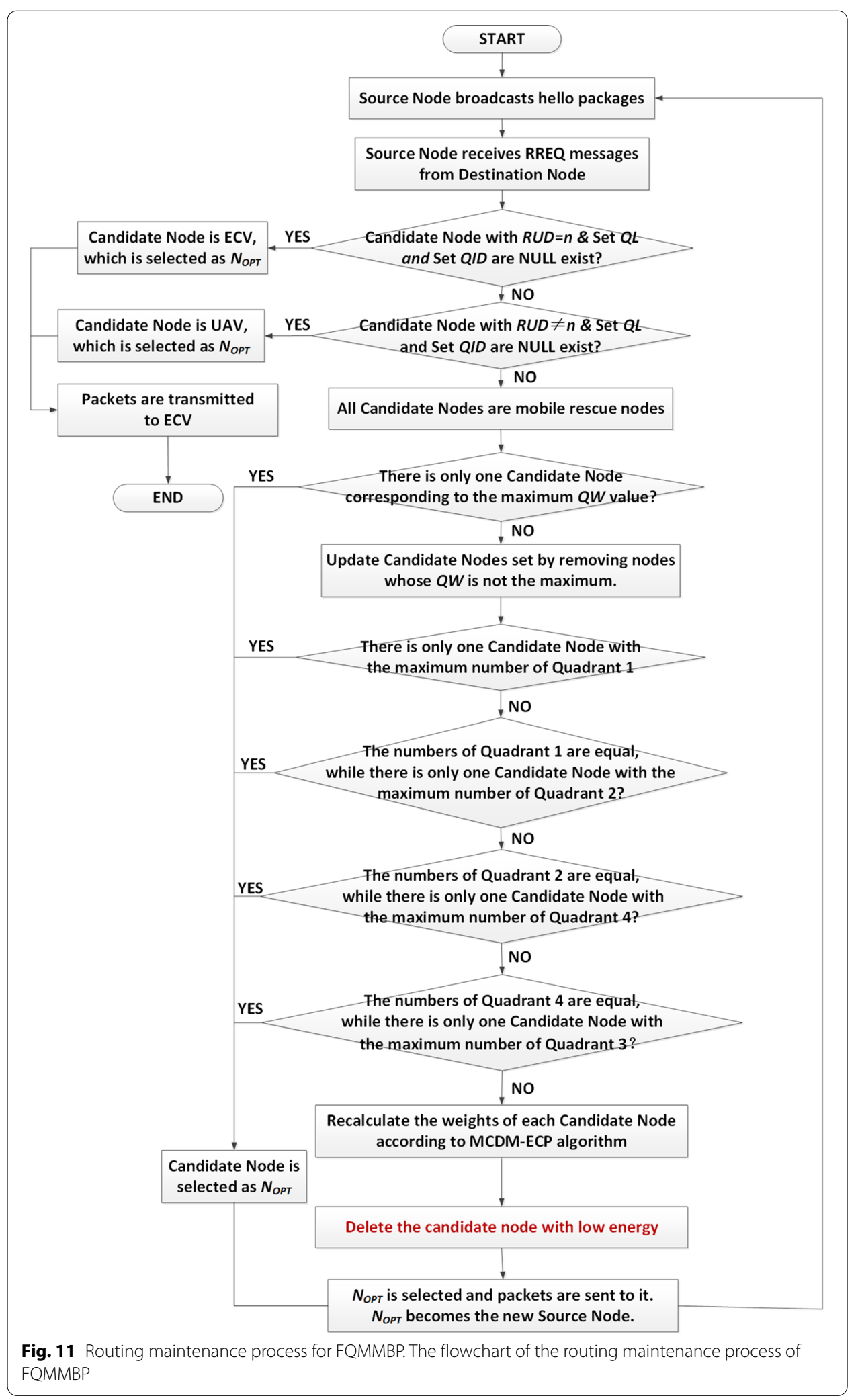


ECV within the coverage of $N_{S}$, then $N_{S}$ determines whether there is a RREQ reply sent by fixed UAV by checking whether there is a candidate node with RUD $\neq n$ and both QL and QID are empty in RREQ reply. If there is an UAV within the coverage of $N_{S}$, data packets are forwarded to UAV, and UAV forwarded data packets to its neighbor. Repeat this process until ECV is founded, finally communication ends. If there are neither ECVs nor UAVs in the candidate nodes, we compare QW values. If there is only one candidate node corresponding to the maximum QW value, then this node is the optimal next hop node (denoted as $N_{\mathrm{OPT}}$ ). $N_{\mathrm{OPT}}$ becomes a new source node $N_{\mathrm{S}}$, and then it sends the hello packets to repeat the process. If the maximum QW value corresponds to more than one candidate nodes, we should update candidate nodes set by removing nodes whose QW is not the maximum. Moreover, the protocols flowchart adds a judgment module to take the energy consumption into consideration. The model will test the candidate relays' remaining energy and selects the suitable candidate as best relay to achieve the communication so that the ECNs which remain the fewer energy will be weeded out from the candidate set to guarantee the quality of rescue. After that, we select $N_{\mathrm{OPT}}$ from the new set according to the following Cases:

Case 1: There is only one candidate node with the maximum number of Quadrant 1. This node is selected as $N_{\mathrm{OPT}}$.

Case 2: The numbers of Quadrant 1 are equal, while there is only one candidate node with the maximum number of Quadrant 2. This node is selected as $N_{\mathrm{OPT}}$.

Case 3: The numbers of Quadrant 2 are equal, while there is only one candidate node with the maximum number of Quadrant 4 . This node is selected as $N_{\text {OPT }}$.

Case 4: The numbers of Quadrant 4 are equal, while there is only one candidate node with the maximum number of Quadrant 3. This node is selected as $N_{\text {OPT }}$.

Case 5: Recalculate the weights of each candidate node according to MCDM-ECP algorithm [22] and then select $N_{\mathrm{OPT}}$.

The description of FQMMBP routing maintenance process is completed.

\subsection{An example}

In order to understand the entire processes of our propose FQMMBP protocol clearly, we give an example to illustrate the main processes including the disaster area division, the main mobility in different RUD, and the relay selection in communication link based on FQMM.

As shown as Fig. 12a, we give an example of a disaster area of $6 \mathrm{~km}^{*} 4 \mathrm{~km}$. According to the degree of emergency importance, the $1 \mathrm{~km} * 2 \mathrm{~km}$ core of earthquake will be divided as $R U D=1$, which means the mobile rescuers will do the task in this area firstly. Based on the equation forehead Sect. 2.2, we can determine and calculate the length and width of each RUD. Besides RUD $=1$ area is rectangular, all the areas of RUD $>1$ are rectangular rings. Based on the results of disaster area division, the smaller RUD id will be rescued earlier. For this instance, we divide 3 disaster areas, RUD $=1$ will be rescued first. $R U D=2$ area is rescued once the tasks in $R U D=1$ area are finished. So are the tasks in area of RUD $=3$.

As shown as Fig. 12b, we suppose that 50 rescuers are set into the disaster area. When $\mathrm{RUD}=1$, the shape of area is rectangular, we first divide the area into four quadrants. 


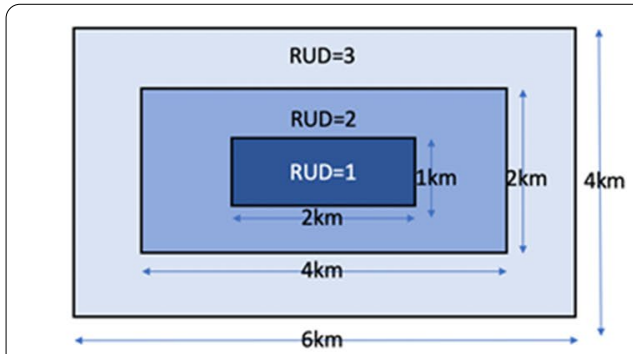

(a) disaster area division

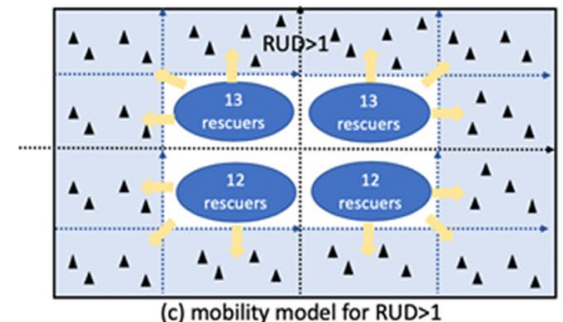

(c) mobility model for RUD>1

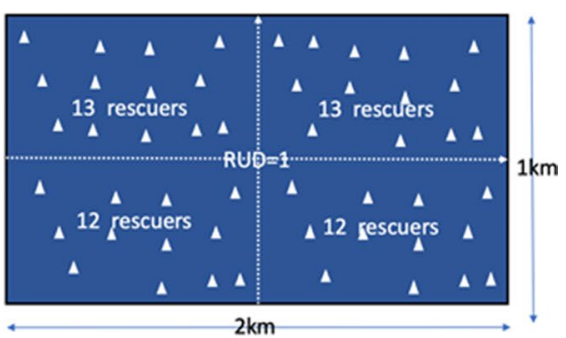

(b) mobility model for RUD $=1$

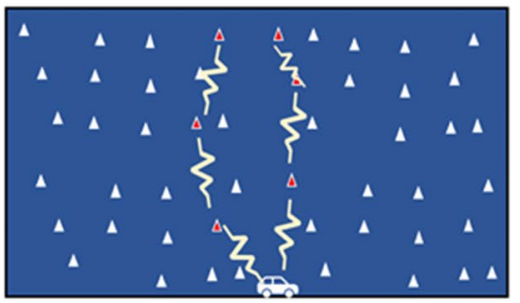

(d) communication link based on FQMM

Fig. 12 The example for entire processes of FQMMBP. An example to explain the whole process of FQMMBP, including the area division idea, the rescuers' mobility in both RUD $=1$ and $R U D>1$, and the communication routing idea during the mobility

According to the formulae in Sect. 2.3.1 $50 \bmod 4=2$. Therefore, the remaining 2 rescuers is arranged into first and second quadrants. As a result, 50 rescuers are arranged into four quadrants as 13,13,12 and 12 rescuers.

As shown as Fig. 12c, RUD $=2$ and $R U D=3$ are the same rectangular rings, which the rescuers are focused in one quadrant of the whole sub-quadrants. For the upper 2 subquadrants, $13 \bmod 3=1$. Therefore, 13 rescuers are arranged to the other 3 quadrants as 5,4 and 4 rescuers. For the lower 2 sub-quadrants, $12 \bmod 3=0$. Therefore, the 12 rescuers mobile into the other 3 quadrants evenly. Attentionally, the rescuers must mobile and begin the next RUD's tasks after the last RUD's tasks are finished. And the additional one rescuer is often arranged to the quadrants that are nearly to the central line.

As shown as Fig. 12d, the communication link is setup from the communication vehicle that located at the rim of disaster area to the rescuers in four quadrants. The core communication link goes throughout the central line of disaster area so that the conditions of all quadrants can be acquired. Based on the formulae in Sect. 2.4, every rescuers as a sequence to identify its own position in the quadrants. The routing protocol will select the suitable relay nodes, as shown as red triangular in the figure to realize the communication to rescue vehicle. As cluster head, these relay nodes will also collect the real-time traffic and rescue information in their own sub-quadrants.

\section{Results and discussion}

In this section, we present the network level performance evaluation and simulations results of the proposed routing protocol based on NS-2 platform. For comparison, with the respect to each relevant class of routing protocols, we selected the pertinent protocols which are the most widely used (e.g., AODV[19], DSR[20], DSDV[20]). 
Table 3 Simulation parameters

\begin{tabular}{ll}
\hline Parameters & Values \\
\hline Number of mobile rescue nodes $(M)$ & 48 \\
Fixed communication nodes (ECV included) & 3 \\
Simulation time span & $600 \mathrm{~s}$ \\
Area of earthquake affected regions & $10 \mathrm{~km} * 10 \mathrm{~km}$ \\
$a_{1}$ & $6 \mathrm{~km}$ \\
$b_{1}$ & $4 \mathrm{~km}$ \\
\hline
\end{tabular}

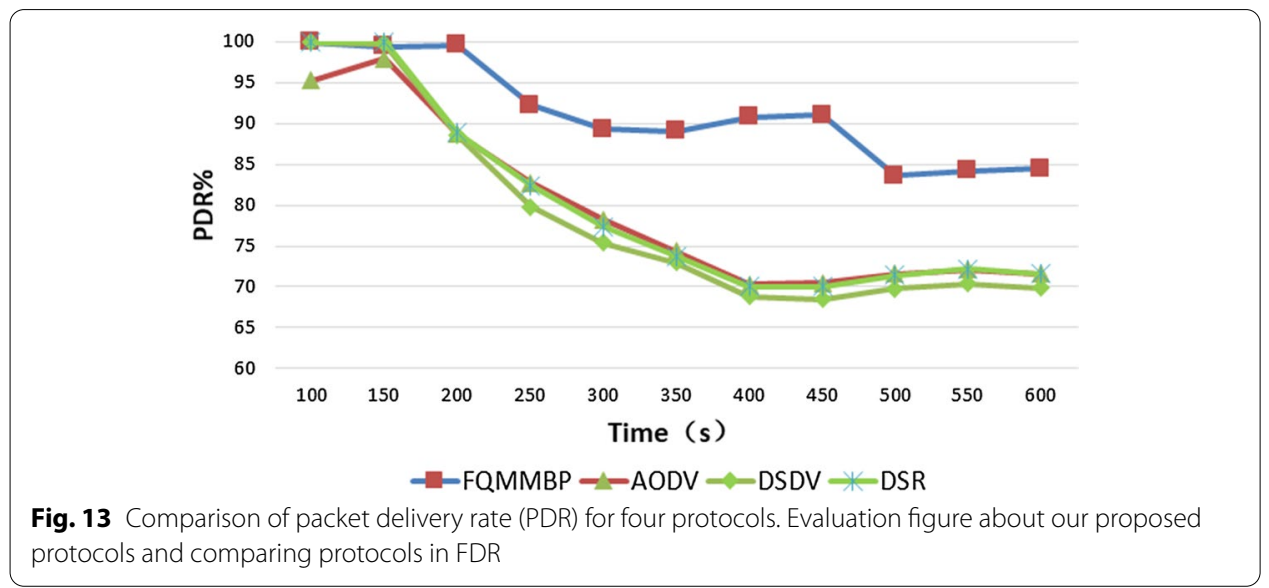

\subsection{Experiment setup}

The simulation environment is a $10 \mathrm{~km} \times 10 \mathrm{~km}$ earthquake disaster area, in which the epicenter of the most severely affected area is $6 \mathrm{~km} \times 4 \mathrm{~km}$. There are 48 mobile rescue nodes, two fixed UAVs and one ECV. The simulation setup and respective parameters are detailed in Table 3.

\subsection{Experiment results}

In this section, four routing protocols, e.g., FQMMBP, AODV, DSDV and DSR, are analyzed from the perspective of package delivery rate (PDR), end-to-end delay (delay) and overhead.

\subsubsection{Package delivery rate}

Figure 13 shows the performance of Package Delivery Rate (PDR) for the different studied protocols. Our proposed FQMMBP achieves the best performance with nearly 91.24\% of average PDR while the other three protocols with about $80 \%$ of average PDR. This proves that FQMMBP improves communication efficiency and stabilizes communication link status in a long period of time. DSDV achieves the worst performance with $79.23 \%$ of average PDR mainly due to the mobility of communication nodes. DSDV is a proactive protocol, and each node maintains a route table. In the emergency rescue scenario, routing tables are dynamic due to the mobility of rescuers, thus causing the cost of maintaining routing tables. The superiority of FQMMBP over other three routing 
protocols is mainly attributed to FQMM mobility model. In each disaster area under this model, the distribution of rescue mobile nodes tends to be uniform, which increases the probability that each node can find the appropriate next-hop node to forward data and ensures the integrity and reliability of communication link. Packets are likely to be delivered successfully in such distributions of nodes.

Compared to other protocols, our proposed FQMMBP has the best performance in FDR, which is increased to AODV, DSDV and DSR as $15.81 \%, 17.59 \%$ and $15.54 \%$, respectively. Therefore, our proposed routing protocol can improve average FDR as $16.31 \%$. And when the simulation time denotes $450 \mathrm{~s}$, our FQMMBP has the best increments in FDR compared to DSDV, which rises $32.87 \%$. However, the FDR performance of FQMMBP at the beginning of simulation has the fewer advantage, because the rescuers should move into the core area of earthquake rather than the rim of scenario, which the FDR will be weak.

PDR performances of the four compared protocols are almost the same before $150 \mathrm{~s}$, that is because all nodes are concentrated in the central area with RUD $=1$ at the beginning of simulation. At this point, the distributions of nodes in the four protocols are similar, and each node has not started to disperse or move to the next area according to the mobility model. As time goes on, the PDR of each protocol decreases gradually. This is because when the nodes complete the rescue tasks in the central area, they will spread to the next peripheral rectangular ring, resulting in the scattered distribution of nodes and the increase in distance between nodes, so the stability of communication link becomes poor. On the contrary, mobile nodes are evenly distributed and close to ECV under our proposed FQMMBP, which improves PDR.

\subsubsection{End-to-end delay}

Figure 14 shows the performance of End-to-end delay (delay) for the different studied protocols. In terms of delay, FQMMBP is significantly lower than other classical routing protocols. During the simulation, the average delay decreased from 1.44 $\mathrm{s}$ of DSR to $0.55 \mathrm{~s}$ of FQMMBP, which proves the significant improvement of communication performance. This is because in each rescue area, the distribution of mobile nodes tends to be uniform under FQMMBP, which increases the probability that each node can find the

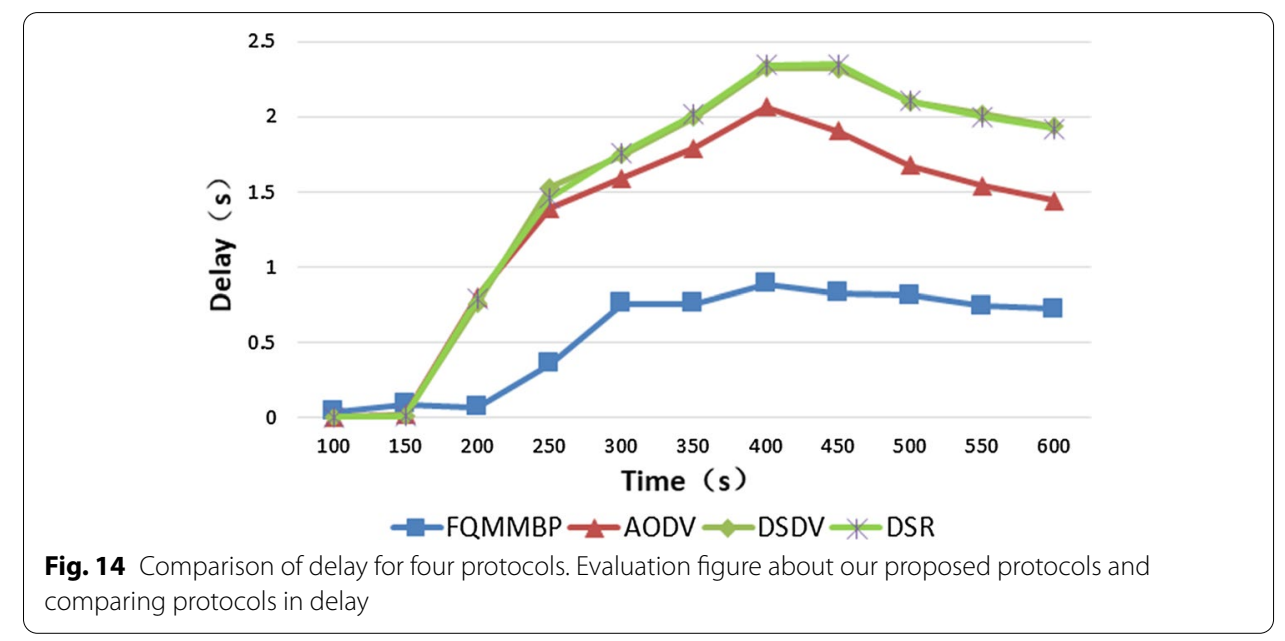


next hop node to communicate with. Within the coverage of each relay node, the existence probability of candidate next hop node increases, which shortens the time to find the candidate next hop node as well as the time to transmit data to ECV.

Compared to other protocols, our proposed FQMMBP has the best performance in delay, which is reduced to AODV, DSDV and DSR as $60.20 \%, 66.56 \%$ and $66.58 \%$, respectively. Therefore, our proposed routing protocol can decrease average delay as $64.45 \%$. And when the simulation time denotes $400 \mathrm{~s}$, our FQMMBP has the most decrement in delay compared to DSR, which is $1.459393 \mathrm{~s}$. The reason of the trend of FQMMBP at the beginning is also above the rescuers' mobility.

Performances of four compared protocols are almost the same before $150 \mathrm{~s}$. This is because at the beginning of the simulation, all nodes are concentrated in the central rectangular area with the most serious seismic intensity. At this time, the distributions of nodes are relatively centralized and similar, and the nodes have not started to disperse and move according to their respective mobility models, so the performance differences of the four protocols are not significant. As time goes on, the delay of each protocol increases gradually, because all nodes will spread to the next peripheral rectangular ring when the rescue tasks in the central area are completed. The dispersion of nodes leads to the increase in distance between nodes; therefore, it takes longer for relay nodes to find the appropriate next hop nodes as the decreasing numbers of candidate next hop nodes. The distribution of mobile nodes is more uniform under the proposed FQMMBP, which shortens the delay.

In the period of $200-300 \mathrm{~s}$, the delay increases obviously as the nodes spread to the next area around when they finish the rescue tasks in the central area. The node distribution changes from the original rectangular centralized to the rectangular ring decentralized, so the node distribution density becomes low, which increases the delay. After $300 \mathrm{~s}$, the delay tends to be stable. This is because there is no significant change in the distributions of nodes when they move from the area with RUD $=2$ to the areas with RUD $>2$. In that case, the delay tends to be stable because of the little impact on FQMMBP.

\subsubsection{Overhead}

Figure 15 shows the performance of overhead for the different studied protocols.

In terms of overhead, FQMMBP is significantly larger than other classical routing protocols. That is because four new fields (RUD, QL, QID and QW) are added to RREQ message. Furthermore, the dispersion of nodes increases the number of forwarding packets, which also contributes to the increase in overhead. The data transmission overhead of AODV, DSDV and DSR protocols increases with time, while FQMMBP meets the same rule from 100 to $300 \mathrm{~s}$. However, the overhead of FQMMBP is decreasing between 300 and $450 \mathrm{~s}$, that is because before $300 \mathrm{~s}$, the rescue nodes are distributed centrally, and they are far away from the destination nodes. Most of the data transmissions rely on fixed relay nodes for forwarding, so the overhead is increasing. In the period of 300-450 s, the distribution of rescue nodes is scattered, and the average distance between rescue nodes and destination nodes is close. In that case, the number of data forwarding is reduced, so the overhead is reduced. After $450 \mathrm{~s}$, the distribution of 


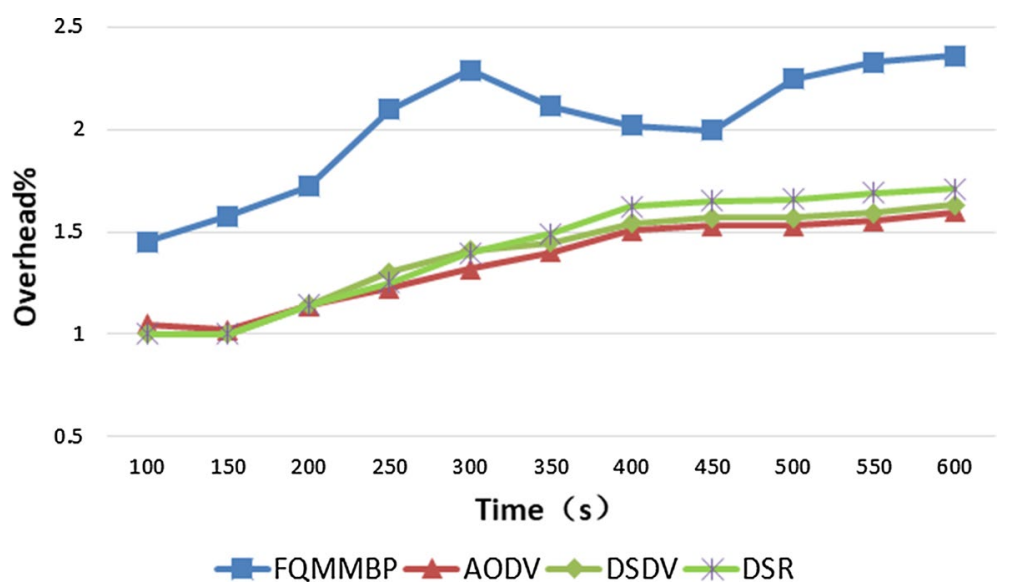

Fig. 15 Comparison of overhead for four protocols. Evaluation figure about our proposed protocols and comparing protocols in overhead

rescue nodes is more scattered than before, and the data communication between nodes relies on multi-hop forwarding, which makes the data transmission overhead increasing. After $600 \mathrm{~s}$, the overhead of FQMMBP can be controlled within 2.5\%.

As a negative impact of lower delay and higher FDR, our FQMMBP lost some extents of overhead, which is a metric relating to the energy and rescuer's cost. Compared to other protocols, our proposed FQMMBP has increased overhead to AODV, DSDV and DSR as $49.89 \%, 46.83 \%$ and $43.92 \%$. Our proposed routing protocol has average $46.88 \%$ increased overhead. We trade $46.88 \%$ overhead loss to gain the benefits of $64.45 \%$ delay and $16.31 \%$ FDR. It is a remarkable and acceptable results for the specific earthquake scenario, which the victims' life is much more important than rescuers' battery. Although the performance of FQMMBP in overhead is not as good as the other three protocols, it is worthwhile for the emergency rescue.

\subsection{Discussions}

\subsubsection{Energy consumption}

There is no doubt that energy consumption is an important metric in mobile rescue issue. In our previous work, we have analyzed the energy consumption during the communication and mobility [23].

Because of the revised model and flowchart, we also evaluate them in the simulation. The simulation results show that the best relay selection is not influenced by the adding energy judgment module. Because the relay candidate is not changed, we added the discussion about this issue in this sub-section, rather than input the same simulation results figure in order to make the manuscript brief. The reason has two points. One is that the magnitude of energy consumption. Comparing the energy consumption during the rescue task, the energy consumption in ECNs, in other words, in the wireless communication is much less. The most part of energy consumption of rescuer is the process of rescue task, rather than the communication. The other is that the previous version considered the overhead, which has the mapping relationship with energy consumption. Therefore, the previous relay selection results are the same as the current improved 
selection results. We also added the discussion about this in the overhead simulation results analysis.

Overall in two sides, the energy consumption is taken into consideration in our previous work, and the energy exhaustion problem can be solved in the realistic emergency rescue scenario.

\subsubsection{Performance metrics selection}

We have two anticipations for our proposed protocols: one is that the ECNs can be set up as soon as possible, which is related to the metric of delay. This metric (delay) can guarantee the rescuers acquire and collect the real-time information about the disaster and victim with short time. The other is the communication link can maintain as well as possible, which means the ECNs can acquire the sufficient information and related to the metric of FDR. For this work, all selected performance metrics (delay and FDR) are useful for the victims in the earthquake. For general scenario, energy consumption is a vital metric for communication modules. The lower energy consumption, the longer modules can remain. However, in this specific earthquake scenario, the lives of victims (human) are much more important than the modules' (electric rescuers). We should guarantee the human being's life with the fast (lower delay) and successful (high FDR) information, not one communication module's duration in the communication link. The life of human is more vital and indispensable than the life of communication module. Actually, in order to save victims lives, we scarify ECN's energy as the addition cost. This is our primary reason to select the delay and FDR as metrics, rather than energy consumption. Moreover, based on our revision, which took the energy consumption into consideration, the protocol's selection results are not changed.

\subsubsection{Routing protocols selection}

In our previous work's simulation results, the selection policy has the lowest energy consumption. Therefore, we improve the selection policy by considering the rescuers' mobility model and update it as FQMMBP. The idea of disaster area's division, and the four-quadrant mobility model are our main contribution about the proposed routing protocol. This protocol has two advantages: one is that the protocol inherits the previous lower energy consumption advantage. The other is that the protocol can guarantee the quality of rescue in earthquake because of the disaster area division and rescuers' mobility model.

The other routing protocols cannot work well in the earthquake emergency scenario. DSDV is adapted from the conventional Routing Information Protocol (RIP) to ad hoc networks routing. It adds a new attribute, sequence number, to each route table entry of the conventional RIP. Using the newly added sequence number, the mobile nodes can distinguish stale route information from the new and thus prevent the formation of routing loops. DSR is set up with the route discovery and route maintenance mechanism to make sure that the packages can be set to the destination node when the source lack the information about the destination node's grouping ID. AODV is a table-driven routing protocol, which will set up the routing table first and then search the effective relay node in the table to transmit the package. The routing protocols above have the relative good performance in MANET and VANET. However, they cannot fit the earthquake emergency scenario. 
Because these routing protocols do not consider the disaster areas' distribution character and mobility model's influence on the rescue quality.

\title{
4 Conclusion
}

Routing protocols for post-earthquake emergency communication network are different with traditional ones because of the heterogeneity and dynamicity of the network. After the earthquake, rescue urgency degree is related to the affected areas. In this paper, we first divide the whole disaster area into several regions with different RUD values according to catastrophic intensity obtained. A four-quadrant mobility model (FQMM) for rescuers based on RUD is proposed. With FQMM, the most seriously affected areas are rescued as soon as possible, furthermore, the rescue crews are allocated to each appropriate quadrant, which improves the rescue efficiency and shortens the time of rescue. Under this mobility model, we propose the FQMMBP protocol for emergency communication network, which improves the RREQ message by adding four new fields: RUD, QL, QID and QW.

A virtual earthquake scenario is simulated, and results show that FQMMBP is superior to traditional routing protocols (AODV, DSDV and DSR) in performances of PDR and Delay. We trade $46.88 \%$ overhead loss to gain the benefits of $64.45 \%$ delay and $16.31 \%$ FDR. It is a remarkable and acceptable results for the specific earthquake scenario, which the victims' life is much more important than rescuers' battery.

In our further work, the model will focus on the global rescue, which means that one task may be finished by several rescuers. Once one rescuer exhausts out, the nearby rescuers can receive this information based on the ECNs in this paper and continues the remaining rescue task, which depends on the optimization model.

\begin{abstract}
Abbreviations
ECV: The emergency communication vehicle; BS: Base station; UAV: Unnamed aerial vehicle; RUD: Rescue urgency degree, which is inversely proportional to the seismic intensity value in this area. The closer the area is to the epicenter, the lower the value of RUD is, which means the disaster is serious and the priority of rescue is high; Otherwise, the opposite. When RUD =1, the epicenter disaster area is a rectangle; When RUD > 1, the disaster areas are rectangle rings that expands outwards in turn; $a_{i}$ : Length of area where RUD $=i ; b_{i}$ : Width of area where RUD $=i ;$ QL: Quadrant Level, which is the number of times the quadrant divided; QW: Total weight of the node located in Qid; QID: Quadrant ID; RT: Number of rescue tasks; NRA: Number of rescuers allocated; $W_{\mathrm{QID}}$ : Weight of QID; $D_{\mathrm{QID}}$ : Distance between the center of each quadrant and ECV; M: Number of mobile nodes.
\end{abstract}

\section{Acknowledgements}

We would like to thank Ping Li who guided and gave us constructive suggestions at the beginning of the research. This research was funded by China Earthquake Administration under Grant No.XH21009. The preparation of this paper has also received support from Shanghai Sheshan National Geophysical Observatory under Grant No.2020Z04, Technology Commission of Shanghai Municipality under Grant No. 18DZ1200500, and Shanghai Sailing Program under Grant No.21YF1432800.

Authors' information

Xiaoming Wang: received his M.S. degree from Lanzhou University, Lanzhou, China, in 2008. He is currently working as a senior engineer in Shanghai Earthquake Administration; meanwhile, he is working towards his Ph.D. degree in Intelligence Communication in Donghua University, Shanghai, China His research interest includes vehicular networks, sensor networks and emergency communications.. Chang Guo: received the B.S. degree in Communication engineering from Donghua University in 2014 and Ph.D. in Information and intelligent communication system from Donghua University in 2020. Her research interests include the data delivery delay in VANETs, the traffic information acquisition, short-term traffic flow prediction, real-time path planning and load balance optimization in urban area.

\section{Authors' contributions}

XW contributed on the work design, acquisition and analysis of data, and drafted the manuscript. CG did the works on the design of model and algorithms, and revision the manuscript based on the comments. All authors read and approved the final manuscript. 


\section{Funding}

This research was funded by China Earthquake Administration under Grant No.XH21009. The preparation of this paper has also received support from Shanghai Sheshan National Geophysical Observatory under Grant No.2020Z04, Technology Commission of Shanghai Municipality under Grant No. 18DZ1200500 and Shanghai Sailing Program under Grant No.21YF1432800.

Availability of data and materials

Data sharing not applicable to this article as no datasets were generated or analyzed during the current study.

\section{Declarations}

\section{Competing interest}

The project is partially funded by China Earthquake Administration, other parts are paid directly by projects partners. One of the main aims of the activities is the generation of UAV's FQBMMBP routing protocol. So, it is given to publish all the main funding in the project to force standardization in this area.

\section{Author details}

'Shanghai Earthquake Administration, Shanghai, China. ${ }^{2}$ Shanghai Sheshan National Geophysical Observatory, Shanghai, China. ${ }^{3}$ The College of Information, Mechanical, and Electrical Engineering, Shanghai Normal University, Shanghai, China. ${ }^{4}$ Shanghai Engineering Research Center of Intelligent Education and Bigdata, Shanghai Normal University, Shanghai, China.

Received: 4 July 2020 Accepted: 15 June 2021

Published online: 26 June 2021

\section{References}

1. Y. Hu. Earthquake engineering in China. Earthq. Engin. Engin. Vib. 1, 1-9 (2002). https://doi.org/10.1007/ S11803-002-0001-5.

2. W. Decai, N. Sidao, L. Jun, Research status of rapid assessment on seismic intensity. Prog. Geophys. 28(4), 1772-1784 (2013). https://doi.org/10.6038/pg20130418

3. W. Yanyan, S. Baiqing, Dynamic multi-stage allocation model of emergency materials for multiple disaster sites. Chin. J. Manag. Sci. 27(10), 138-147 (2019). https://doi.org/10.16381/j.cnki.issn1003-207x.2019.10.014

4. Z. Shuwen, L. Can, Z. Kejun et al., Optimal scheduling strategy with emergency rescue team's characteristics taken into consideration. Manag. Rev. 31(2), 225-237 (2019). https://doi.org/10.14120/j.cnki.cn11-5057/f.2019.02.020

5. Z. Xiaolin, C. Changding, Multi-objective optimization of marine emergency resource dispatching. Navig. China 42(1), 56-62 (2019). https://doi.org/10.1007/s13198-017-0648-y

6. S. Ye, S. Yinghua, L. Dan et al., Earthquake emergency rescue team's assignment model based on time satisfaction and competence. China Saf. Sci. J. 28(8), 180-185 (2018). https://doi.org/10.16265/j.cnki.issn1003-3033.2018.08.030

7. Y. Jinsha, M. Zi, Y. Hong, Research on intelligent dispatching of emergency materials in the early stage of earthquake relief. Sci. Technol. Eng. 20(21), 8702-8708 (2020). https://kns.cnki.net/kcms/detail/detail.aspx?dbcode=CJFD\& dbname $=$ CJFDLAST2020\&filename $=$ KXJS202021041\&V=7ufNVsW8Ho8jt0GPrsuVpNY44URoBpykgoqUDTEcTpGSt OXF9jlqWoJuQyGGYuZ7

8. M.I. Talukdar, M.S. Hossen, Selecting mobility model and routing protocol for establishing emergency communication in a congested city for delay-tolerant network. Int. J. Sens. Netw. Data Commun. 8(1), 1-9 (2019). https://doi. org/10.4172/2090-4886.1000163

9. C. Cejun, L. Congdong, Q. Ting et al., A bi-level programming model for relief trans-regional scheduling: taking into consideration survivors' perceived satisfaction and risk acceptability. J. Manag. Sci. China 22(9), 113-128 (2019). https://kns.cnki.net/kcms/detail/detail.aspx?dbcode $=$ CJFD\&dbname=CJFDLAST2020\&filename $=J C Y J 201909$

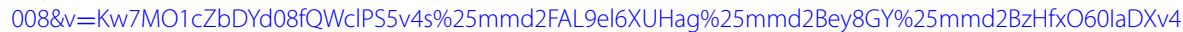
cstr\%25mmd2FmU

10. S. Yinghua, G. Yan, D. Lijing et al., Study on location and distribution of emergency facilities considering the psychology of victims. J. Catastrophol. 34(1), 187-193 (2019). https://doi.org/10.3969/j.issn.1000-811X.2019.01.034

11. Y. Ruilin, Positioning of wireless sensor network under emergency communication environment. Instrum. Mesure Métrol. 19(4), 273-279 (2020). https://doi.org/10.18280/i2m.190404

12. Li. Guangqiang, He. Jia, A routing algorithm based on the probability of encounter between nodes in delay-tolerant network. Comput. Era 01(01), 33-36 (2021). https://doi.org/10.16644/j.cnki.cn33-1094/tp.2021.01.008

13. Y. Xiang, T. Siyu, X. Xin, The adaptive routing algorithm based on emergency communications of TD-LTE. Wuxian Hulian Keji 21, 1-4 (2015). https://doi.org/10.3969/j.issn.1672-6944.2015.21.001

14. M. Bamhdi Alwi, Efficient dynamic-power AODV routing protocol based on node density. Comput. Stand. Interfaces (2020). https://doi.org/10.1016/j.csi.2019.103406

15. M. Syed Rabiya, R. Ramalakshmi, Replica reduced routing protocol for intermittent connected networks in emergency scenarios. Int. J. Distrib. Syst. Technol. (IJDST) 10(2), 84-109 (2019). https://doi.org/10.4018/IJDST.2019040105

16. F. Masud, A.H. Abdullah, G. Abdul-Salaam, Emergency traffic adaptive MAC protocol for wireless body area networks based on prioritization. PLoS ONE (2019). https://doi.org/10.1371/journal.pone.0225518

17. R. Ramalakshmi, S. Radhakrishnan, Weighted dominating set based routing for ad hoc communications in emergency and rescue scenarios. Wirel. Netw. 21(2), 499-512 (2015). https://doi.org/10.1007/s11276-014-0800-4

18. V. Nivedhitha, A.G. Saminathan, P. Thirumurugan, DMEERP: A dynamic multi-hop energy efficient routing protocol for WSN. Microprocess. Microsyst. 79, 103291 (2020). https://doi.org/10.1016/J.MICPRO.2020.103291 
19. V. Bondre, S. Dorle, Performance analysis of AOMDV and AODV routing protocol for emergency services in VANET. Eur. J. Adv. Eng. Technol. 4(4), 242-248 (2017)

20. B. Ramakrishnan, R.B. Nishanth, M.M. Joe, M. Selvi, Cluster based emergency message broadcasting technique for vehicular ad hoc network. Wirel. Netw. 23(1), 233-248 (2017). https://doi.org/10.1007/s11276-015-1134-6

21. T. Camp, J. Boleng, V. Davies, A survey of mobility models for ad hoc network research. Wirel. Commun. Mob. Comput. 2(5), 483-502 (2002). https://doi.org/10.1002/wcm.72

22. X. Wang, D. Li, X. Zhang, Y. Cao, MCDM-ECP: multi criteria decision making method for emergency communication protocol in disaster area wireless network. Appl. Sci. 8(7), 1165 (2018). https://doi.org/10.3390/app8071165

23. X. Wang, D. Li, C. Guo, X. Zhang, S.S. Kanhere, K. Li, E. Tovar, Eavesdropping and jamming selection policy for suspicious UAVs based on low power consumption over fading channels. Sensors 19(5), 1126 (2019)

\section{Publisher's Note}

Springer Nature remains neutral with regard to jurisdictional claims in published maps and institutional affiliations.

Submit your manuscript to a SpringerOpen ${ }^{\circ}$ journal and benefit from:

- Convenient online submission

Rigorous peer review

- Open access: articles freely available online

- High visibility within the field

- Retaining the copyright to your article

Submit your next manuscript at $\mathbf{s p r i n g e r o p e n . c o m ~}$ 